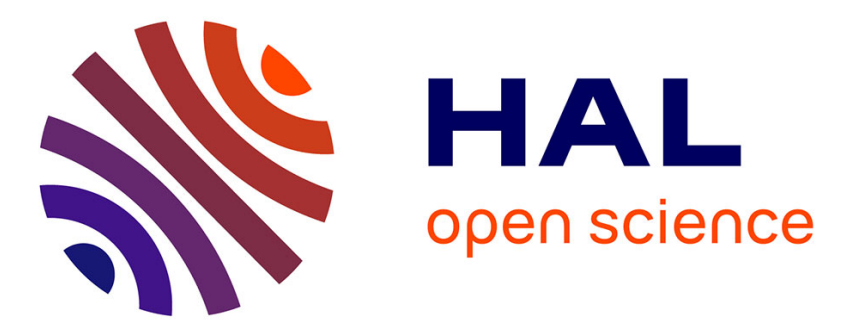

\title{
Décors sur sigillée dans le style de Paternus II : moules et gobelets Déch. 68
}

Colette Bémont

\section{To cite this version:}

Colette Bémont. Décors sur sigillée dans le style de Paternus II : moules et gobelets Déch. 68. Gallia - Fouilles et monuments archéologiques en France métropolitaine, 1975, 33 (1), pp.171-202. 10.3406/galia.1975.1518 . hal-01937297

\section{HAL Id: hal-01937297 \\ https://hal.science/hal-01937297}

Submitted on 11 Mar 2020

HAL is a multi-disciplinary open access archive for the deposit and dissemination of scientific research documents, whether they are published or not. The documents may come from teaching and research institutions in France or abroad, or from public or private research centers.
L'archive ouverte pluridisciplinaire HAL, est destinée au dépôt et à la diffusion de documents scientifiques de niveau recherche, publiés ou non, émanant des établissements d'enseignement et de recherche français ou étrangers, des laboratoires publics ou privés.

\section{(이) $\$$}

Distributed under a Creative Commons Attribution - NonCommercial - NoDerivatives $\mid 4.0$ 


\title{
DÉCORS SUR SIGILLÉE DANS LE STYLE DE PATERNUS II : MOULES ET GOBELETS Déch. 68
}

\author{
par Colette BEMONT
}

Un article récent de Grace Simpson, dans la série que l'éminente spécialiste anglaise consacre aux vases noirs métallescents du C'entre de la Gaule ${ }^{1}$, vient de renouveler l'attention pour un style décoratif encore peu étudié et attribué - - en particulier à la suite des travaux de J. Déchelette ${ }^{2}$ - à l'atelier de Palernus II de Lezoux. L'intérêt du fragment publié en Angleterre ${ }^{3}$ tient principalement au répertoire, où les motifs géométriques occupent une très large place, et à la structure de la frise, dont les panneaux, séparés par des torsades, s'organisent, au moins une fois sur deux, selon une symétrie bilatérale. Or cet exemplaire appartient à un groupe en grande partie inédit, dont la coll. Plicque, au Musée des Antiquités nationales, contient plusieurs échantillons : des moules et des vases, dont les frises ont comme caractéristiques communes essentielles un mobilier iconographique associé, pour sa plus grande part, au style de Palernus II et une organisation en métopes séparées par des cordons torsadés.

Il m'a paru utile, puisque le tri des moules conservés au musée de Saint-Germainen-Laye m'avait conduite à rassembler ce matériel, de le micux faire connaitre. J'ai cherché, d'abord, à définir les caractères d'une série de matrices, homogène à la fois par les décors et par la qualité et le profil des pièces. La comparaison avec un ensemble de fragments de vases, noirs ou rouges, a permis ensuite d'étendre la liste des poinçons utilisés pour ces gobelets, de préciser différents modes d'organisation des frises et d'étudier les relations de ce groupe avec d'autres échantillons de formes ovoïdes - en particulier un vase célèbre, mais que la publication de J. Déchelette, malheureusement approximative, a tant soit peu déformét. Enfin, en me référant autant que faire se pouvait à des exemplaires signés,

1 More black slip vases from Central (jaul with applied and moulded decoralion in Brilain, dans The Antiquaries Journal abrégé A.J. dans la suite de l'article, I.III, 1973, p. 42-51.

2 Vases céramiques ornés de la Gaule romaine, Paris, 1904, vol. I, pl. IV a p. 152.

3 o. c., p. 49, fig. 1,39 et p. 50, s.39. Gobelet trouvé à lincoln en 197l, dans un contexte non daté.

4 La reproduction des dessins de louvrage de Déchelette dans la publication de J.A. Staxminto et G. Simpsox, Central gaulish polters, Oxford, 195x abrégé S.S., pl. 56 profil at 104, 1 frise, éclaircil limage originale, un peu floue, du décor, mais en accentue légerement certaines inexactitudes. 


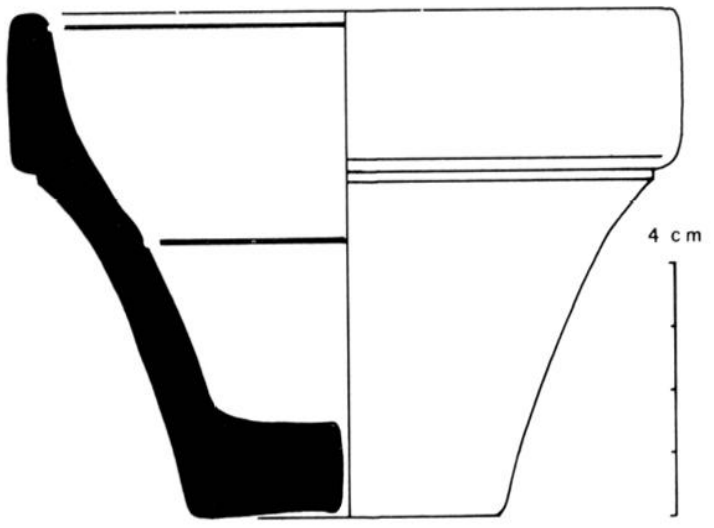

I Profil diun moule : P.I 224.

souvent inédits, j'ai donné un état des points communs entre cet outillage décoratif et celui de Palernus II et j'ai tenté de montrer, à l'aide de quelques exemples, la parenté des différentes compositions ornementales avec colles que l'on voit sur des formes plus classiques issues soit du même atelier, soit d'autres, proches de lui.

La série des matrices comprend les fragments de quatorze pièces dont le diamètre interne supérieur varie entre environ 9,5 et $15 \mathrm{~cm}$ : neuf échantillons se situent entre $11, \overline{5}$ et $12,5 \mathrm{~cm}$, quatre entre 9,5 et $10,5 \mathrm{~cm}$, un seul atteint probablement $15 \mathrm{~cm}^{5}$. Tous les moules, faits d'une pâte rosâtre, granuleuse, très micacée, le plus souvent assez cuite, appartiennent à un mème type - - de forme globalement tronconique, pourvu en sa partie supérieure d'un épaississement formant un large bandeau --, qui rappelle assez certains pots à fleurs actuels (fig. 1). Tout le groupe, également, comporte, avec de légères variations (et à condition que l'état le l'ohjet permette d'en juger), certaines particularités qui, dans la collection du musée, ne se trouvent régulièrement associées que sur ces échantillons: l'obliquité, plus ou moins prononcée, du bandeau, son profil - rectiligne pour l'essentiel, arrondi en sa partie inférieure ${ }^{6}$... et la concavité accentuée de la panse. Sans présumer de l'étroite spécificité de cette morphologie, il importe de la décrire, dans la mesure où sa régularité semble correspondre à des décors également homogènes, toujours par leur répertoire, souvent par leur organisation.

Les frises ressortissent apparemment à dleux systemes principaux (fig. $8, A$ et $B$ ). L'un est illustré sûrement une fois, peut-être deux : il consiste en un découpage du décor en métopes identiques aménagées de la même façon. Le second, attesté certainement, ou probablement, par les douze autres matrices, est fondé sur l'usage alterné de deux panneaux différents l'un de l'autre. C'est dans cette série nombreuse que se place le vase publié par Grace Simpson. Le premier type de structure apparait sur un moule entièrement décoré de panneaux occupés, chacun, par un arceau habité, au-dessus d'un motif construit sur

5 P.1 321. Lablat de conservation du fragment ne permet quine mesure approximative el decourage loule tentalive d'orientation precise de lobliquite de la panse.

6 I. méplat qui souliene lallache du bandeau a la panse ne peut en toule rigureur être considere comme un de ces caracteres associes : il manque sur deux echantillons 321.45 el PM 2:31 la friabilite de la surface aulorise à supposer que l'usure peut expliquer la disparition du meplat, sil a jamais existe, mais on doit conslater son absence et la serie est trop courte pour quion nighige ce fait.

7 P.I 22.4 a P.I 3:1. 
une symétrie bilatérale (fig. 2, PM 224). Un autre échantillon, malheureusement très petit, comporte, de part et d'autre d'un cordon torsarlé sommé d'un lleuron, les restes de deux cercles lisses apparemment de mème taille (fig. 2), P.I 3221). Ces maigres vestiges autorisent à imaginer hypothétiquement soit une frise entièrement ornée de médaillons, soit un rythme d'alternance entre des métopes différentes qui permette la succession de deux panneaux semblables. Onze des douze autres pièces ont conservé au moins la trace d'une séquence constituée d'une métope pleine et d'un panneau contenant un arceau en sa partie supéricure (fig. :2 et :3). Le dernier ${ }^{8}$, trop petit et mal situé pour qu'on puisse observer cet enchaînement, contient, du moins, des fragments de l'arceau et l'indication d'un registre inférieur (fig. 3, P.I 230). Aussi est-on fondé, compte tenu de la similitude des poinçons, de la taille de l'objet et de la remarquable monotonie du reste de la série, à supposer qu'il appartenait au même groupe que les onze moules précédents. Aucune des matrices les mieux conservées ne permet de concevoir autre chose qu'une composition uniformément fondée sur la quadruple répétition de la suite de deux métopes précitée ${ }^{9}$ et les petits fragments dont on peut restituer le décor, gràce à la taille des motifs utilisés ${ }^{10}$, font apparaître, au moins, que la succession (partiellement conservée) de deux panneaux correspond à un quart de la longueur de la frise. On relève, dans l'organisation de détail, deux genres de variantes : la métope pleine "st occupée tantôt par un sujet figuré libre, tantôt par un médaillon habité, les vides chant, dans l'un et l'autre cas, comblés par des poinçons de remplissage. D'autre part, le second panneau, qui comporte deux registres séparés ${ }^{11}$ ou non par un cordon horizontal, selon la place laissée par l'arceau en bas de la frise - est meublé le plus souvent ${ }^{12}$, en sa partie inférieure, d'une composition à symétrie bilatérale : deux éléments identiques se répètent simplement ${ }^{13}$ ou s'opposent de part et d'autre d'un décor vertical14, généralement un astragale (fig. 2, 3, 5). Cependant, deux échantillons rompent plus ou moins avec cet usage : P.I 231 (fig. 3) présente, à la place de cet agencement, un poinçon unique, une colonne couchée à deux tambours semblables; le fragment P.I 225 (fig. 2) exclut toute symétrie puisqu'il conserve un fragment d'un motif orienté -- la tête d'un poisson nageant vers la gauche par ailleurs bien connu ---, qui doit suffire à meubler toute la largeur de la métope.

La combinaison rythmée des poinçons, enfin, dans la mesure où une assez longue séquence permet d'apprécier leur périodicité, ne témoigne pas d'une particulière recherche : seul le moule 32412 (fig. 3) conserve, dans les arceaux de deux séquences successives, deux oiseaux semblables, orientés l'un vers la droite, l'autre vers la gauche, et non le même volatile uniformément répété. En ce qui concerne le répertoire attesté par cette série, un inventaire rapide donnant, avec l'état du moule, soit le contenu du morceau de frise, soit, dans les meilleurs cas, le mobilier d'une séquenere, permettra d'estimer ce matériel.

$8 \quad$ P.II 230.

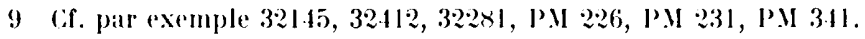

10 Ainsi sur P.II 225, P.II 227, P.II 229.

11 Moules 32145, P.II 225, P.II 227, P.M $2331-232$.

12 Six fois $32145,322 \times 1,32412$, 1.11 226, P.11 229, P.11 3.11 sur huil echantillons conservant le registre inferieur.

13 l'est ce qu'il advient dans lr cas des roselles.

14 Gest une possibilite quoffrent des poincons a ș̣etrie hilaterale : les palmes ot les fleurons peuvent etre

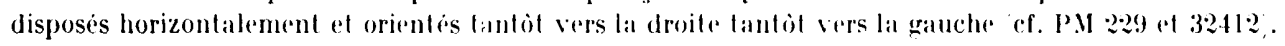




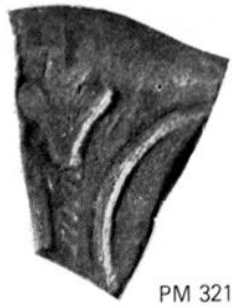

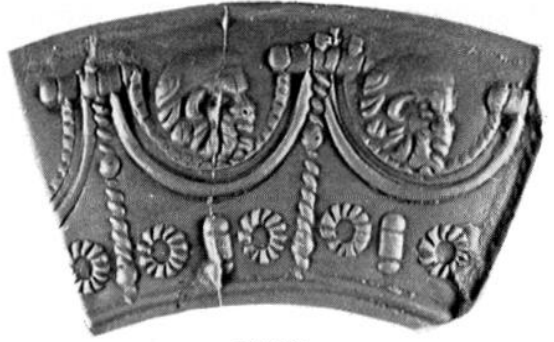

PM 224

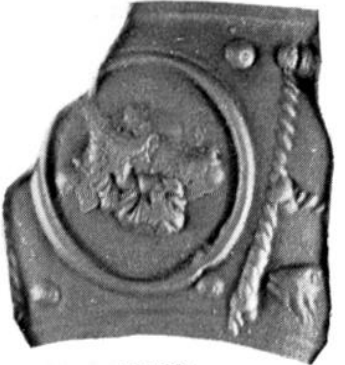

PM 225

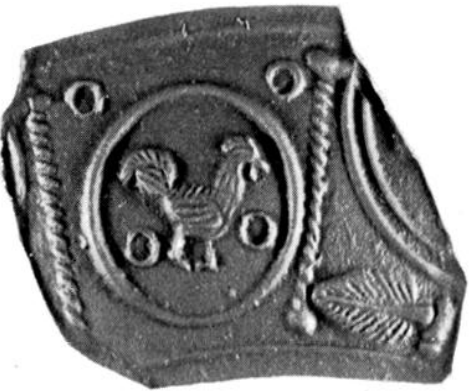

PM 229

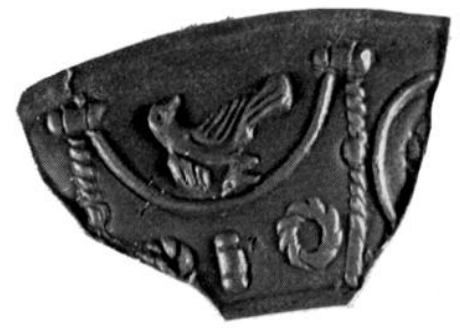

PM 226

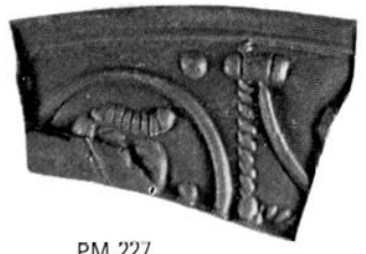

PM 227

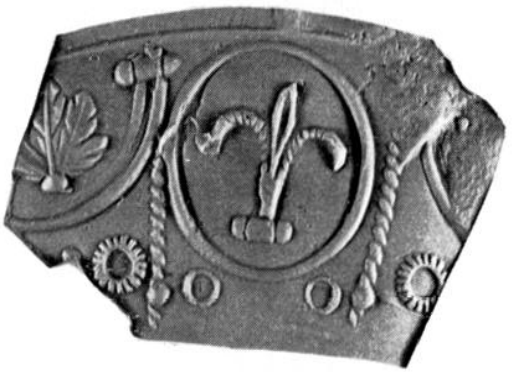

32281

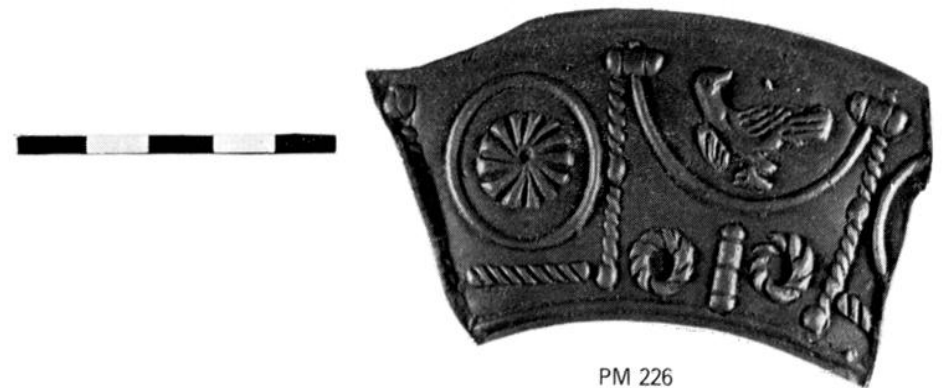

PM 226

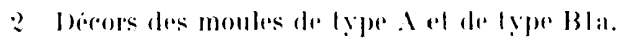

Type $A^{15}$ (fig. 2 et 8 )

PM 224. o $10 \mathrm{~cm}$, haut. 8 et $6,5 \mathrm{~cm}^{16}$; profil entirement conservé ; décor composé de huil panneaux identiques dont six et demi subsistent. Poinçons ${ }^{17}$ : 1 cordon $\mathrm{A}: 366^{18}, 29$ astragale $\mathrm{R}$ 60, 15 pois ; métope: 21 petite rosette à douze pétales obliques et cour largement évidé, même astragale, 7 double arceau composé d'un arc extérieur lisse, d'un arc intérieur strié transversalement, $4 \tau$ masque de Silene à droite I) $694=01341$.

15 Ie type A rassemble les frises decoupres an melopes identiques. Il semble ici comporter derax variantes : l'une, certaine, a arceaux, l'autre, probable cf. infra, n. 37, i médaillons, mais le nombre des echantillons esl trop, fatible, leur qualitie, trop inegalle, pour justifier une classification plus joussiee.

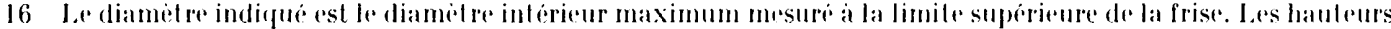
correspondent respectivement aux mesures exterienre ol interienre du moule sauf dans les cas, daalleurs signales, où seule la hauteur interieure est conservere.

17 Les cordons, communs aux panneaux qüils siparent, ainsi fue les poincons qui ponctuenl, lerminent ou articulent ces séparations pois, rosettes, astragales, fleurons sont dabord enumeres, puis le contenu de chague métope : le même motif, selon sa place, peul donc être mentionne deux ou plusieurs fois. lees chiffres qui precedent le nom ou la definition de chaque poincon renvoient aux nos de mon catalogue tableaux I at II, fig. 9.

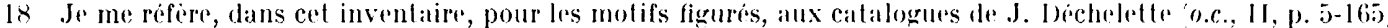
et de F. oswald Index of Figne lypes on Terra sigillala, Liverpoul 1936/1937, abreges respectivement 1) et (), et pour las motifs non figurés, au travail de (x. B. Rogers l'oleries sigillées de la liaule cenlrale. I. Les molifs non figurés, XXVIle supp. à Ciallia, 1975, à lamilie de qui je dois d'avoir pu consulter le manuscrit de louvage. 
P.M 321. " env. 15 ' 'm! : tries petit fragment de la partie supérieure de deux panneaux. I'oinçons: 1 cordon .1 36, 32 fleuron trifide (i 153); métope: 13 cercle lisse $(04 \mathrm{~cm})$.

Type R. Série 1 a: à médaillons ${ }^{19}$ (fig. 2 et 8 )

I'.II 226. 10.5 ('m ; deux fragrments de frise : l'un courre un peu plus du quart de la circonférence et comprend deux métopes complites, l'une a médaillon, l'aulre a arcean ef la partie gauche d'une I roisième apparemment semblable i la première; le second, inférieur au quart de la frise, conserve presque tout un panneau i arceau et la partie gauche d'une métope à médaillon. Poinçons: 1 cordon 136,29 astragale $\mathrm{R}(60,1.5$ pois ; métope pleine: 11 cercle lisse (: 2.5 ('m), 22 grande rosette ¿ treize pétales obliques, 25 barre à neuf torsades descendant de gauche i droite; métope à arcean: mème astragale, 2 arceau lisse $(4.1 \mathrm{~cm}$. 51 oiseau st ylisé i qauche 02295$) .18$ rosette a huit pétales courbes et corur largement évidé.

32281. 10.5 cm ; fragment courant légirement plus du quart de la frise et comprenant plus de la moilié droite d'une métope a arceau, une métope à médaillon el la parlie qauche d'un second panneau ì arceau. Poingons: 1 cordon 1 36. 29 astragale R 60, 1.5 pois ; mélope pleine: 12 cercle

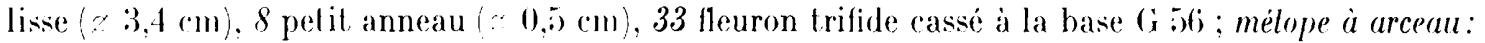
4 petil double arceau lisse ( $\max .4 \mathrm{~cm})$. 24 rouelle a dix-huit dents E :57, 36 feuille découpée II 189. P.II 229. : $11, \overline{5} \mathrm{~cm}$; fragment couvrant un peu moins du quart de la frise et comprenant l'extrémité droite d'une métope à arceau. une métope à médaillon et la partie inférieure gauche d'un second panneau a arreau. Poincons: 1 cordon 1 :36, 30 astragale R 68, 15 pois ; métope pleine: 12 cercle lisse $(\because 3,4 \mathrm{~cm}) .8$ petit anneau $(0,5 \mathrm{~cm}), 52 \mathrm{coq}$ a droite D 1023 $=02342$; métope à arceau: 5 double arceau lisse (s max. $4,6 \mathrm{~cm}) .29$ astragale $\mathrm{R} 60$, 3.5 palme $\mathrm{J} 146$.

I'M 227. " 11,5 cm ; fragment couvrant la partie supérieure de moins d'un quart de la frise et comprenant environ les trois-quarts d'une métope à médaillon et l'extrémité droite d'un panneau a arceau divisé par un cordon horizontal. P'oingons: 1 cordon $A 36$, 29 astrarale $\mathrm{R} 60$, 15 pois ; mélope pleine: 12 (ercle lisse $(33,4 \mathrm{~cm})$, mème astragale, pois, 48 masque cornu à gauche I) $67 \%)=$ () 1214 ; métope à arcean: 3 ? arceau lisse $(z 4,8 \mathrm{~cm} \text { ? })^{20}$.

P.II 22.5. $\approx 11.5 / 12 \mathrm{~cm}$; fragment couvrant environ un huitieme de la frisc et comprenant une métope à médaillon el l'extrémité gauche d'un panneau à arceau divisé par un cordon horizontal. Poinçons: 1 cordon $\triangle 136.29$ astragale R 60,15 pois ; mélope pleine: 12 cercle lisse $(\% 3,4 \mathrm{~cm})$, pois, 47 masque de siline a droite J) $694=0$ (1341: métope à arceau: 3: départ d'un arceau lisse (: 4,8 ('m1?), j3 poisson à gauche I) 1062 = 02417.

Série 1 b: a sujets libres (figr. 3 et 8 )

32412. $11, \bar{c} \mathrm{~cm}$, haul. 9,4 et $8,1 \mathrm{~cm}$; profil entierement conserve; fragment couvrant la totalité

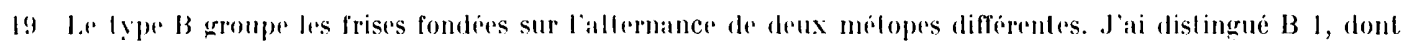
l'une des métopes se différencie de laut pe par la division en deux registres et l'emploi de l'arcean al b, c correspondent a des subdivisions itablies d'apres le contenu de la seconde des melopes, B 2 , dont l'un des panneaux est occupe par un grand medaillon, B 3, qui nutilise aucune des dispositions précedentes. Toutefois, le fractionnement de B 1

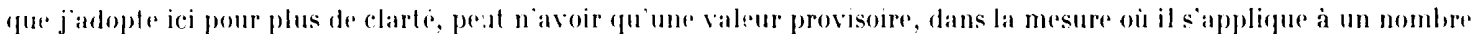
limile dexemplaires, qui me constiturent pas obligatoirement un échantillon significatif. Farmi les pieces dont jai e"u connaissance, les variations qui interessent la melope pleine permettent de distinguer deux groupes principanx

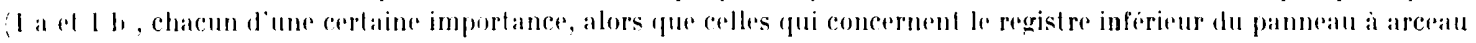

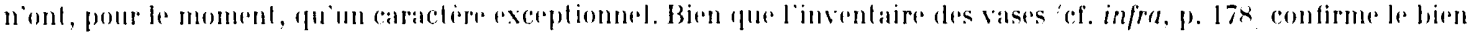

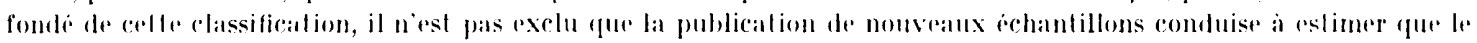

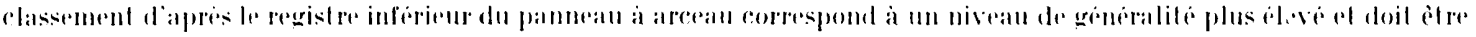

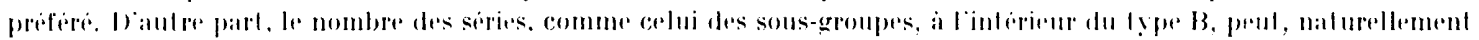
être arecru, si la diversiti du maleriel lexigen.

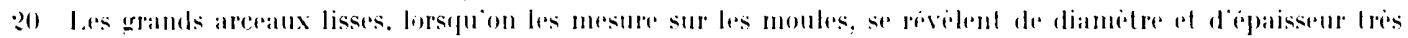

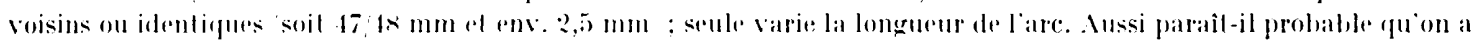
affiale an résultal de l'impression plus on moins complete d'un mème outil. 

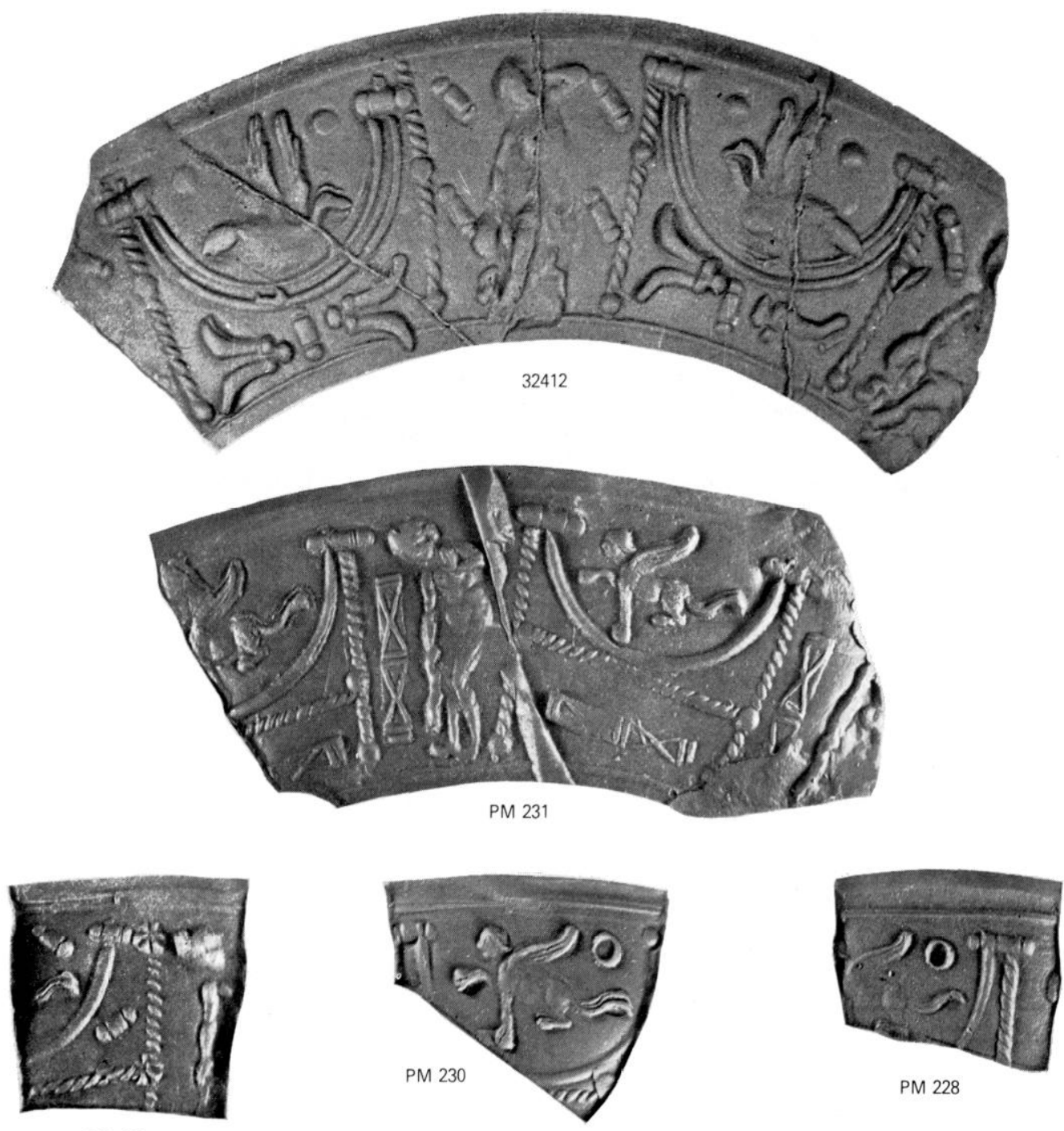

PM 232
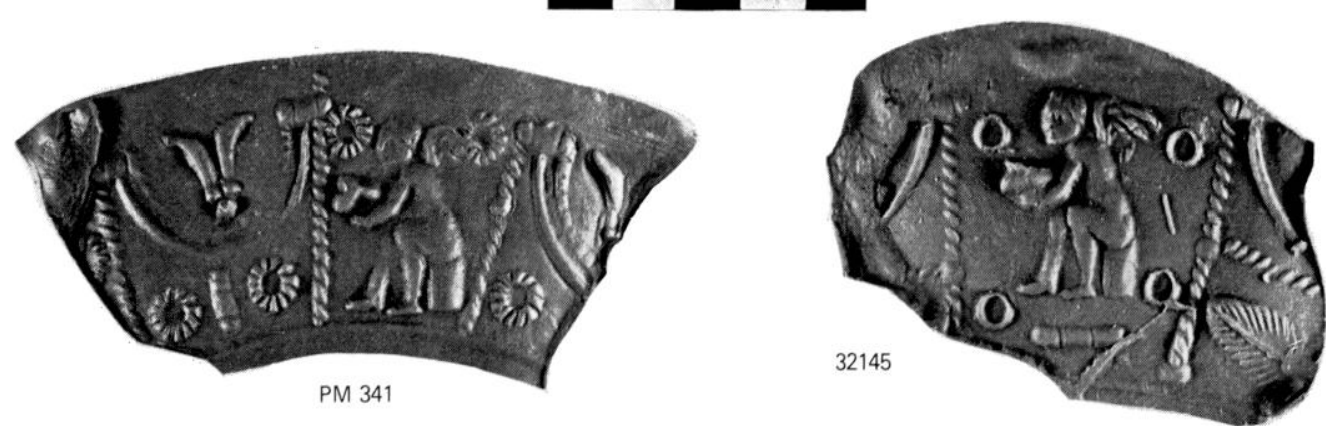

3 Decors des moules de lype BII. 
de la frise à la réserve des deux-tiers d'une métope à arceau. I'oinçons: 1 cordon 136,29 astragale $\mathrm{R} 60,15$ pois ; métope pleine: mème astragale, 46 personnage assis de trois-quarts 0 949; mélope i arceau: $;$ double arceau lisse $(\mathrm{max}, 4,6 \mathrm{~cm})$, mème astragale, pois, 31 fleuron bifide $(\mathrm{s} 335$, 49-50 oiseaux aux ailes éployées alternativement à droite I) 1010 = 0 2316 et à gauche I) $1011=$ 02324.

P.M 341. 9.5 cm, haut. int. $6.4 \mathrm{~cm}$; fragment couvrant plus d'un quart de la frise et comprenant un petit morceau du cóté droit d'un panneau plein (?), une métope à arceau, une métope pleine et la moitié gauche d'un second panneau a arceau. P'oinçons: 1 cordon $\mathrm{A} 336,29$ astragale R 60, 15 pois: mélope pleine: 21 rosette à douze pétales obliques (cf. PII 224), 42 amour assis à gauche 1) $261=0444:$ mélope à arcean: $z$ double arceau intérieurement strié (cf. P.I 224), mème rosette, mème astragale, 31 lleuron bifide (; 33).

32145. $: 11.5 \mathrm{~cm}$; fragment couvrant moins du quart de la frise et comprenant une métope pleine entre deux panneaux à arceau incomplets, divisés par un cordon horizontal. P'oinçons: 1 cordon A 36,29 astragale $R 60,15$ pois ; mélope pleine: 30 astragale $R 68,8$ petit anneau $(x 0,5 \mathrm{~cm})$, 42 amour assis à gauche $\mathrm{I}) 261=0444 ;$ métope à arcecul 3 arceau lisse $(5.8 \mathrm{~cm}), 35$ palme $\mathrm{J} 146$. P.I 231.: 12/12.5 c'm, haut. int. $7.4 \mathrm{~cm}$; fragment courrant environ un tiers de la frise et comprenant quatre métopes alternativemenl coupées avec arceau et pleines, semblables deux ì deux ; le premier des panneaux coupés est mutilé à droile et il ne subsiste que la moitié gauche du serond panneau plein. I'oingons: 1 cordon 1366.30 astragale R 68,15 pois ; métope pleine: 27 colonne à deux lambours barrés, chacun, d'un sautoir, 38 Vénus à sa toilelte I) $181=0$ () 290 ; métope à arceau: 3 arceau lisse (: $4,8 \mathrm{~cm})$. meme colonne, 54 sphinx assis ì gauche J) $497=0857$.

$P . M 232 . \approx 12,5 \mathrm{~cm}$; fragment courrant environ un huitième de la partie supérieure de la frise et comprenant la moitié droite d'une métope à arceau divisée par un cordon horizontal et, probablement, la moitié gauche d'une métope pleine. loinçons: 1 cordon A 36, 29 astragale R 60, 16 rosette à six pétales C 123; métope pleine: 38 Vénus à sa toilette I) $181=0290$; métope $\dot{a}$ arceau: 3 arceau lisse $(: 4,8 \mathrm{~cm})$, même astragale, $\tilde{5} 4$ sphinx assis à gauche D $497=0857$.

P.I 228 . " 11,5/12 ; fragment couvrant la partie supérieure de moins d'un huitième de la frise et comprenant environ les deux tiers droits d'une métope à arceau et l'extrémité gauche d'une métope pleine. Poincons: 1 cordon $\mathrm{A}$ 36, 30 astragale R 68; mélope pleine: 38 Vénus à sa toilette I) $181=0290$ ?; métope à arceau: 3 arceau lisse $(.8 .8(\mathrm{~cm}), 8$ petit anneau $(\% 0,5 \mathrm{~cm}), 54 \mathrm{sphinx}$ assis à grauche I) $497=0$ () 8:57.

Serie $1 b$ ? (fir. . 3)

P.M 230. $11.5 / 12 \mathrm{~cm}$; fragment couvrant la partie supérieure d'environ un huitieme de la frise et comprenant le haut d'une métope ì arceau divisée par un cordon horizontal. Poinçons: 1 cordon A 36. 29 astragale $\mathrm{R} 60$; métope à arceau: 3 arceau lisse $(3,8 \mathrm{~cm}), 8$ petit anneau $(0,5 \mathrm{~cm})$, j4 sphinx assis a gauche I) $497=0$ (0)7.

Parmi les huit fragments de vases inédits ${ }^{21}$ que l'on peut intégrer sûrement ou probablement au mème groupe que les moules, sept sont noirs ou bruns, le dernier ${ }^{22}$, rouge avec des traces noiràtres, couleur due, sans doute, comple tenu des irrégularités, à une réoxydation accidentelle de l'ohjet. Tous ressortissent à la forme Déch. 68. Leur pâte varie du beiga au rose voire au rougeâtre ${ }^{23}$; l'engobe intérieur ou extérieur est métallescent

$\because 1 \quad 3: 2.251,1,59(1-5) \div(6$.

$\therefore 2$ ए

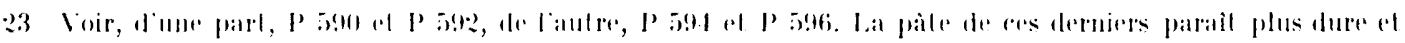
plus cuile. 


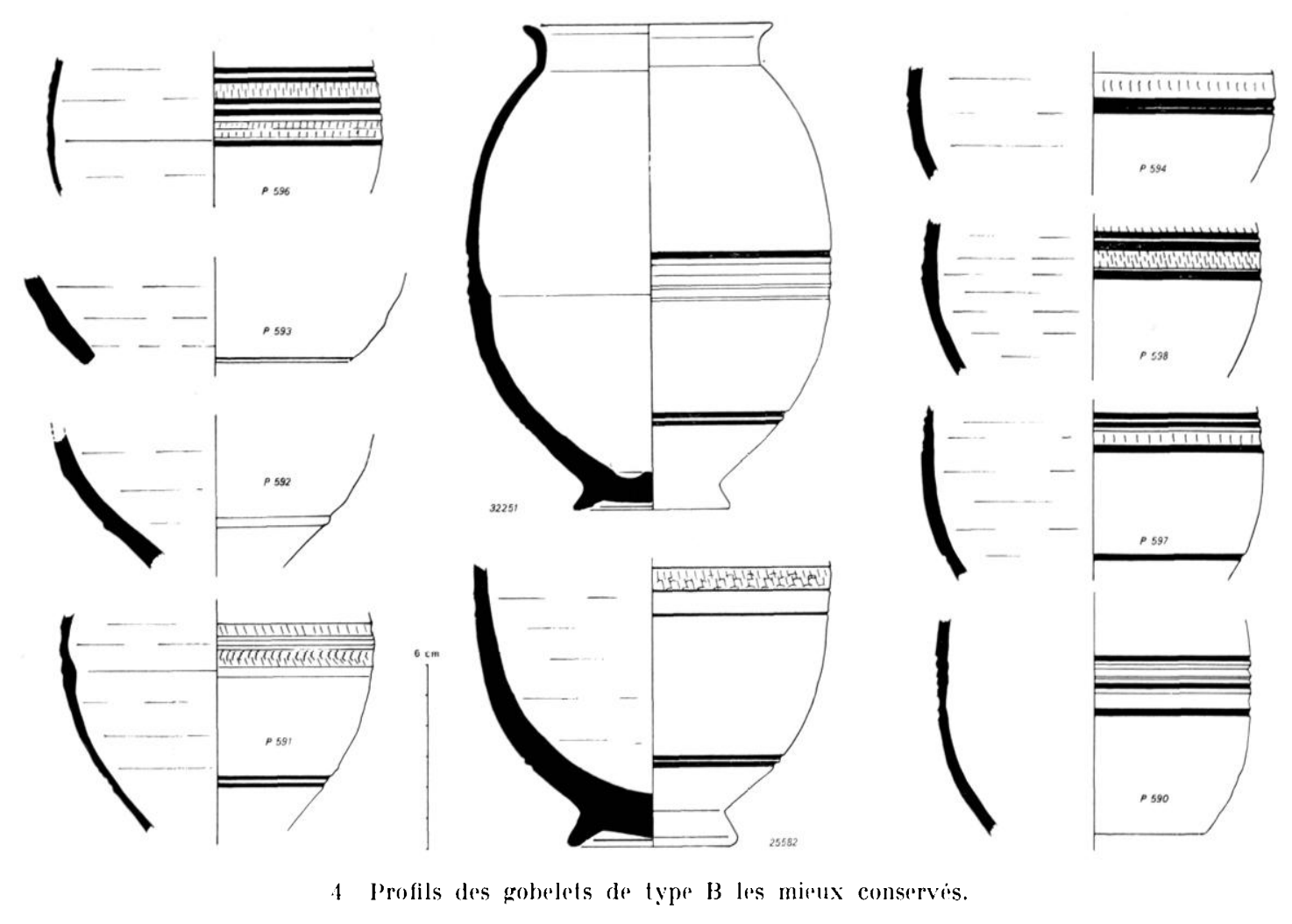

au moins sur six des échantillons noirs. Le dernier d'entre eux, en revanche, un vase presque complet ${ }^{24}$, est terne et très largement écaillé sur les reliefs. Le diamètre des pièces, quand on peut le restituer (fig. 4, 32251, P 590-596) va de 11 à $13 \mathrm{~cm}$, ce qui suppose des moules semblables à ceux que nous venons de décrire, ou d'un module voisin. La structure globale des décors est comparable à celle des douze précédents modèles, les détails de l'organisation, lorsqu'on peut les vérifier, sont, à une réserve près, conformes aux mêmes schémas (fig. 5, 32251, P 590-596). L'inventaire des poincons apporte quelques nouveautés : une brève analyse permettra d'en jugerer.

Type B. Série 1 a: à médaillons (fig. 5 et 8 )

$P 590 . \varnothing \max .11 \mathrm{~cm}$; fragment couvrant, sur presque toute sa hauteur, plus du tiers de la frise et comprenant environ les trois-quarts droits d'une métope pleine, une métope à arceau et la partie supérieure gauche d'un second panneau plein cassé en diagonale. P'oinçons: 1 cordon A 36, 29 astragale $\mathrm{R} 60,15$ pois ; métope pleine: 12 cercle lisse (env. $3,2 \mathrm{~cm}), 48$ masque cornu à gauche D 675$)=01214 ;$ mélope à arceau: 5 double arceau lisse ( $z$ max. $4,4 \mathrm{~cm}$ env.), mème astragale, 11 cercle lisse ( $\approx$ env. $2,4 \mathrm{~cm}$ ), 22 grande rosette à treize pétales obliques, 17 rosette à sept pétales. $P 591 . \approx$ max. $11 \mathrm{~cm}$; fragment couvrant le quart de la frise et comprenant la plus grande partie d'une métope pleine, une métope à arceau et l'extrémité gauche d'un second panneau plein.

2432251.

25 I.'identiticalion des poinçons circulaires ou semi-circulaires, dont le diamétre peul varier selon la qualite du moulage et le retrait de la pate, s'est faite par référence aux poincons bien individualisés que renfermaient ces cadres. On peut constater, d'ailleurs, que la rétraction de l'argile, si l'on compare la taille des mêmes motifs sur moules et sur vases, parâ̂t assez faiblo 'souvent inférieure à $10 \%$ \% 
Poincons: 1 cordon A 36,30 astragale $\mathrm{R} 68,1$.5 rosette ou pois? ; métope pleine: 12 cercle lisse ( $z$ env $3,1 \mathrm{~cm}$ ), 49 oiseau aux ailes éployées à droite $\mathrm{D}) 1010=02316$. petit anneau? ; métope à arceau: 5 double arceau lisse $(z$ max. env. $4,3 \mathrm{~cm})$. même astragale, 22 grande rosette à treize pétales obliques, 37 feuille découpée II 71 .

P 592. $\varnothing$ max. 11,5/12 cm ; fragment courrant un peu plus du huilième de la frise et comprenant le quart droit d'une métope pleine et presque toute la surface d'un panneau à arceau. Poinçons: 1 cordon $A 36,30$ astragale $\mathrm{R} 68,15$ pois : métope pleine: 12 cercle lisse $(\not x$ env. $3.2 \mathrm{~cm}):$ métope à arceau: jouble arceau lisse (cf. supra) mème astragale, pois, 33 fleuron trifide (i 56,18 rosette a huit pétales courbes et cœur largement évidé.

I 594. $\approx$ max. $13 \mathrm{~cm}$ env. ; petit fragnent courrant environ un huitieme du haut de la frise et comprenant l'angle supérieur droit d'une métope pleine et l'angle supérieur gauche d'un panneau

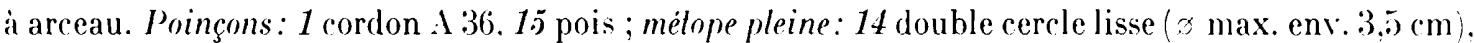
24 rouelle à dix-huit dents ; métope à arceau: 6 double arceau lisse outrepassé ( $\approx$ max. env. 3,5 cm), 49 oiseau aux ailes éployées à droile I) $1010=02316$.

L'état du vase ne permet pas de présumer du contenu du registre sous l'arceau, mais, du moins, le diamètre du double cercle dans le panneau plein autorise-t-il à supposer une plare disponible en bas de l'autre métope.

Série $1 b$ : à sujets libres (fig. j) et 8)

32251. max. $13 \mathrm{~cm}$, haut. $15,8 \mathrm{~cm}$; vase presque entier, dont la frise est composée de quatre séries de deux métopes, l'une pleine, la seconde à arceau et divisée par un cordon horizontal. Poingons: 1 cordon $A 36,30$ astragale $\mathrm{R} 68,15$ pois ; métope pleine: 29 astragale $\mathrm{R} 60,44$ amour debout de face l) $265=0450$; métope à arceau: 3 arceau lisse ( $\not$ env. $4,6 \mathrm{~cm})$, pois, 19 rosette à onze (?) pétales obliques, 25 barre torsadée (cf. PM 226), 49-50 oiseaux aux ailes éployées à droite D $1010=02316$ ou à gauche D $1011=02324^{26}$.

P 596. $\approx$ max. $12 \mathrm{~cm}$; petit fragment couvrant environ un huitieme de la partie supérieure de la frise et comportant toute la largeur d'une métope pleine entre les angles gauche et droit de deux panneaux à arceau. Poinçons: 1 cordon A 36, 29 astragale R 60 ; mélope pleine: 41 épreuve très fruste d'un groupe bacchique I) $307 \mathrm{~b} ?=0561$; mélope à arceau: 5 double arceau lisse (cf. P 591), 21 rosette à douze pétales obliques et cœur largement évidé.

Ce fragment n'autorise évidemment pas à présumer d'autre chose que de l'existence, sous l'arceau, d'une plage disponible.

Série 1 a ou $b$ ? (fig. 5)

1' 595. $\otimes$ ? ; très petit fragment du haut de la frise, comprenant la partie droite d'une métope à arceau et l'angle gauche d'une métope sans arceau. I'oinçons: 1 cordon $A 36,29$ astragale $\mathbf{R} 60$; métope pleine (?): 8 petit anneau ; métope à arceau: 6 double arceau lisse ou outrepassé (cf. P 594), 33 fleuron trifide $\mathrm{G} 56$.

L'exiguïté du tesson interdit toute classification autre qu'hypothétique. On constate simplement l'enchainement de deux panneaux, l'un avec, l'autre sans arceau. Le second ne comportant pas, dans l'angle conservé, de trace de division géométrique, il est possible - comple tenu aussi de la présence du poinçon de remplissage - qu'il ait contenu un sujet libre ou un médaillon.

Deux vases déjà connus se ratlachent à cette série par le principe d'alternance des métopes: un fragment d'ovoüde de forme incertaine, dont il ne reste qu'un morceau de frise - trouvé à

26 les oiseaux nallernent pas, mais sont organises en deux series : les arceaux d'ume moitio du vasu sont occupes par des oiseaux a droile, cenx de laulre moitie, par des oiseaux a gauche. 

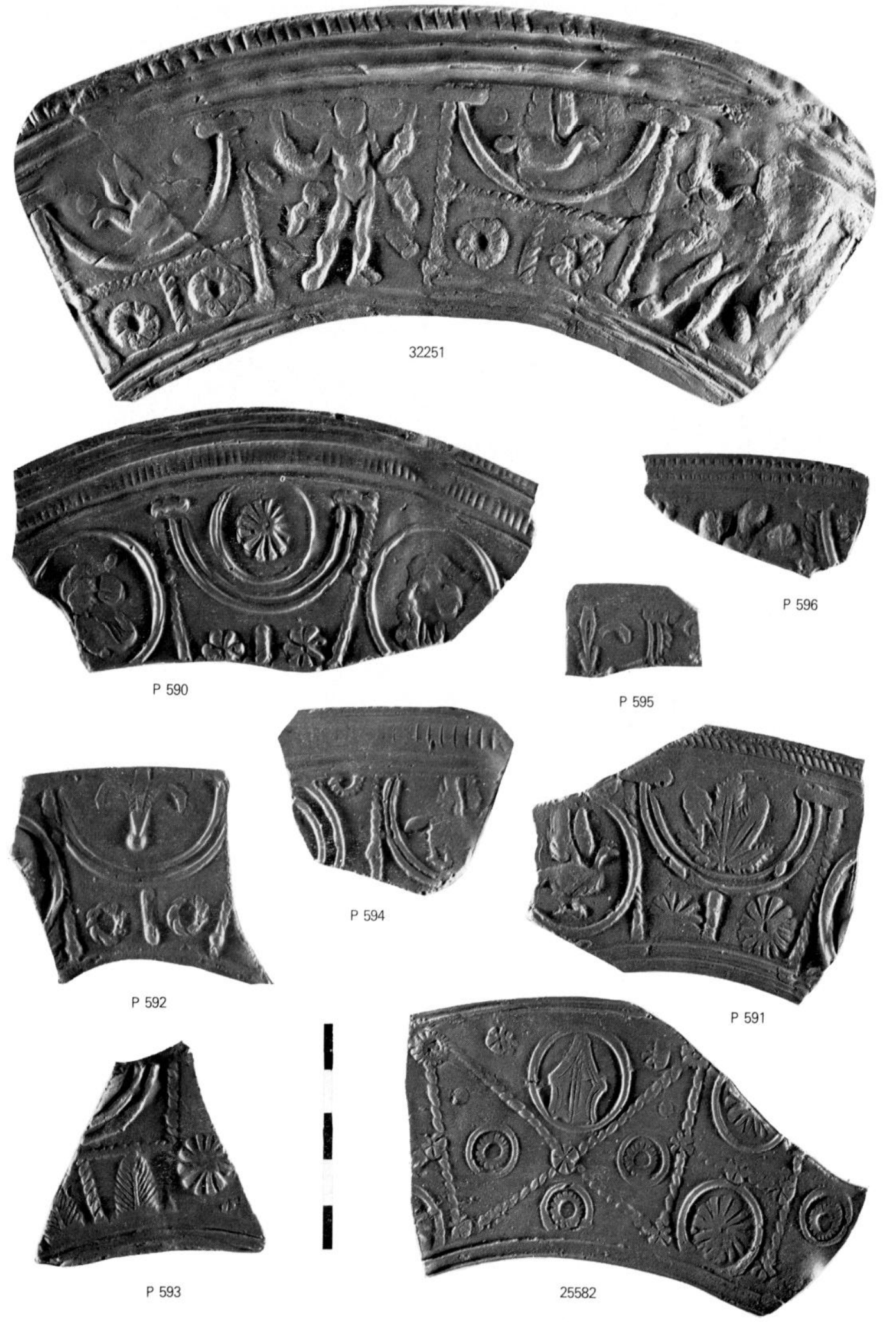

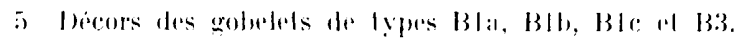


Ilchester, il a été publié en 19572i - et le gobelet l)éch. be de lincoln. déjait cité28. Pour ce dernier, le rapport entre la longueur de frise conservée et le diamitre total. permel sans peine d'imaginer un décor composé de quatre séquences de deux panneaux. Le premier lesson présente sous l'arceau. un poingon orienté, le second, une composilion à trois éléments fondée sur une symétrie bilatérale.

Série 1 c: à dèor géométrique 23 /fig. is et 8 :

I) 593. : max. au moins 13,5) ('m : fragment de la base de la frise couvrant environ un huitime de sa longueur et romprenanl la partie droite d'une mélope à arceau. divisée par un cordon horizontal. et l'angle gauche d'une métope à déror géométrique. Poincons: 1 cordon 1 :36: mélope pleine: mème cordon. 22 grande roselte i l reize pétales obliques: méloper it arcerun: jo double arceau lisse

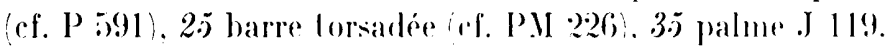

L'inventaire des tessons de vases inédits permet d'ajouter quelques poinçons supplémentaires à la série attestée par les moules ot par les deux gobelets anglais : des rosettes -une à sept, l'autre à onze (?) pétale's . une feuille découpée, un motif figuré, l'amour debout (fig. 9, 17, 19, 37, 44). In groupe en très maurais état, mais dont la silhourette évoque un décor bacehique à trois personnages, pose un petil problème : s'agit-il du motif très fruste déjà connu à Lincoln ou d'une autre variante du mème sujet? La qualité des deux reliefs laisse perplexe : la place respective de la tête des différents personnages ne paraît pas la même sur les rleux gobelets (fig. 9, 41), mais comme le tesson du Yusée des Antiquités nationales ne conserve que la partie supérieure du groupe, on ignore si le mème poinçon n'a pas pu ètre différemment incliné vers la droite ou la gauche ${ }^{30}$.

Autre nouveauté : le dernier de nos tessons de gobelets (P 593) présente, dans le panneau plein un type particulicr d'organisation (fig. 5) et 8, I 1 c), clont un vase de la coll. Rambert aide à reconstituer la structure ${ }^{31}$ : cet échantillon -- attribuable, du fait de son répertoire, au même atelier ${ }^{32}$ que toute notre sério -..- comporte une métope écartelée en sautoir et dont des motifs géométriques occupent les quatre quartiers (fig. i) et 8, B, 3).

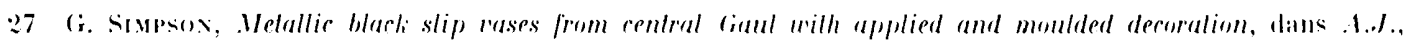

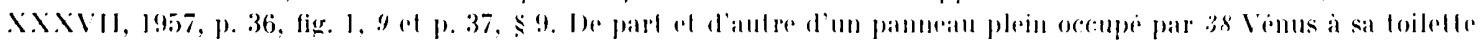

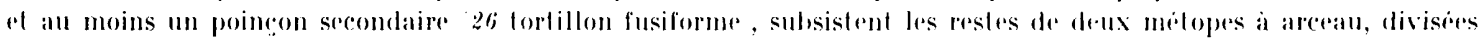

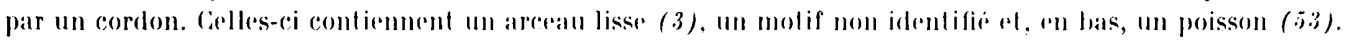

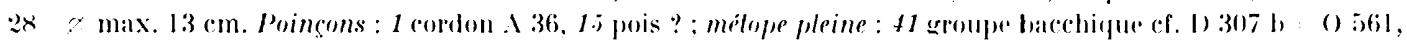

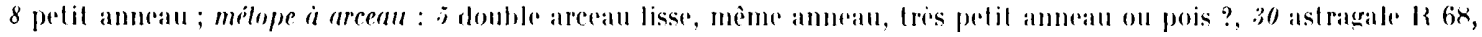

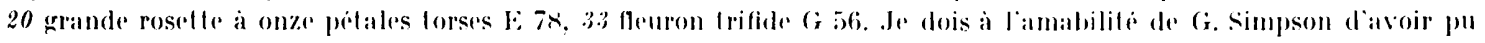
disposer des dessins origrimaux da vaso el de sa frise.

29 lif. n. 19 a infra. p. 193.

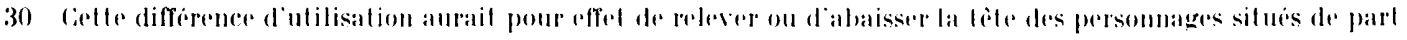
el daute de Bacchus el. lorsegue manque le bas de la frise, de les faire paraitre plus ou moins grands. Il ne semble

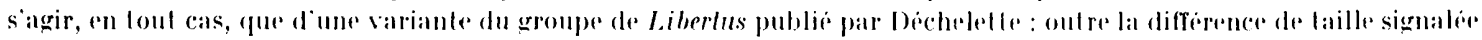

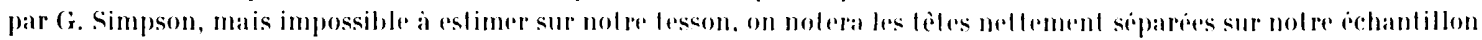

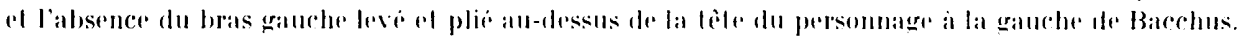

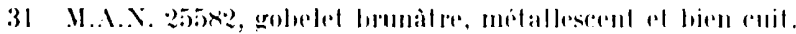

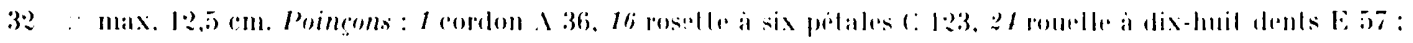

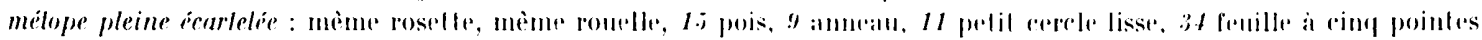

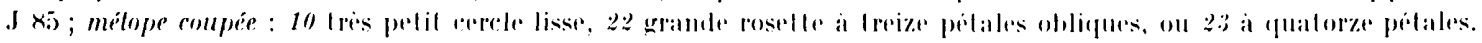

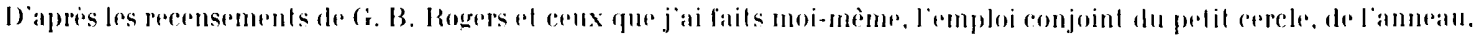

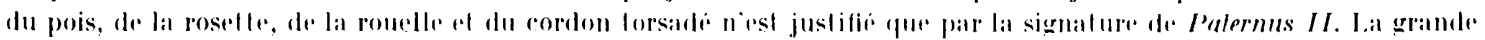

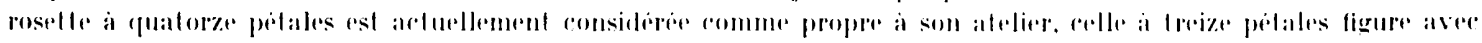

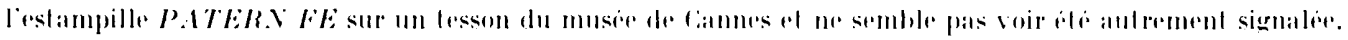


Or, seul un sautoir explique de façon satisfaisante le tronçon de cordon oblique conservé dans la métope pleine de P 593.

Comme on peut le voir, d'après l'inventaire que résume le tableau I, cet ensemble de vingt-quatre décors, comprend vingt-deux pièces construites sur l'alternance certaine ou probable de deux métopes : l'une sans arceau, l'autre avec arceau. Le contenu du panneau plein permet de répartir presque également dix-neuf des frises en deux groupes: les unes contiennent des médaillons, les autres en sont dépourvues. Linfin, sur les vingt-deux échantillons, quinze nous restituent le registre inférieur de la métope à arceau : celui-ci est occupé certainement onze fois, vraisemblablement douze, par un motif à trois éléments et à symétrie bilatérale. Lil série, si l'on se reporte à la fois à ces principes abstraits de classification et aux objets cux-mèmes, témoigne de la monotonie des structures et de la multiplicité des combinaisons possibles entre des poincons, pour la plupart de petite taille. L'invention et l'originalité ne sont donc pas les caractères dominants de cet ensemble. Il serait injustifié, cependant, de ne pas apprécier à sa valeur l'intérêt de ce défaut d'imagination. J'ai eu l'occasion déjà ${ }^{33}$ de montrer comment les principes généraux d'organisation des frises fournissent un moyen de classer des vases dans des groupes géographiques et chronologiques, parce que les mêmes schémas décoratifs se retrouvent, à la même époque, dans les ateliers d'un même centre. Dans le cas présent, l'analyse plus détaillée du matériel permet de mettre en lumière de façon concrète et précise, à l'intérieur d'une officine, la qualité d'un travail en série : un même type de vases, fabriqué avec des moules en tous points comparables entre eux, présente - du fait, entre autres, du module peu varié des pièces -... - un décor fondé sur une structure globale identique; en la circonstance, huit métopes alternent deux à deux et le contenu de chacune d'elles s'organise selon des principes peu variables d'un exemplaire à l'autre.

L'examen des moules et des gobelets aux fins de comparaison fait ressortir un autre caractère de cette production. Tandis que les moules attestent du soin ou de l'habileté du potier par toutes sortes de qualités : modelé des poinçons, régularité de la disposition, finesse de la pâte, cuisson satisfaisante, les vases paraissent déplorablement négligés. On ne peut, évidemment, tenir compte ici des imperfections imputables à la cuisson : la collection du M.A.X. est composée, pour une grande part, de vases de rebut, découverts sur le lieu de fabrication. Du moins doit-on noter que des pièces jugées bonnes pour la cuisson (et accidentellement manquées ou brisées) étaient moulées et finies sans le moindre égard pour le décor : empreintes incomplètes, surimpressions, motifs écrasés, voire partiellement oblitérés, en haut, par le guillochage, en bas, par des gorges creusées au moment de la reprise du pied (figr. 5). Il est avéré, également, que ces défauts ne rendaient pas la vaisselle impropre à la vente : certaines pièces imparfaites ont été exportées ${ }^{34}$. Il

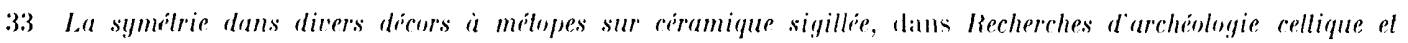

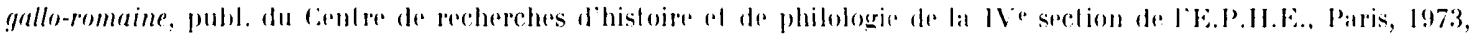

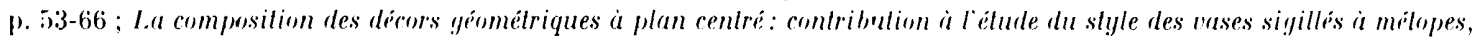

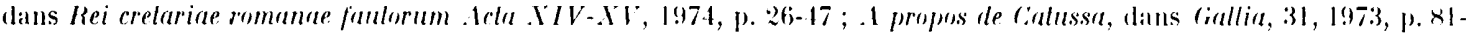
122.

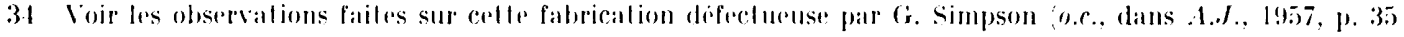
e 37, : " 
paraît illogique, au premier abord, qu'on ait destiné au commerce des objets dont l'ornementation, si banale soit-elle, prouve au moins quelque souci de diversité, dont la qualité des moules témoigne d'un trarail soigné, mais dont la fabrication, elle-même, laisse tant à désirer. Imaginer - l'hypothèse est des plus vraisemblables - que le travail était divisé, que la qualification de la main d'ceuvre pouvait varier notablement selon la spécialité de chaque ourrier et qu'un décorateur adroit risquait d'être trahi par un mauvais potier -surtout si la demande entrainait une accélération néfaste des opérations ${ }^{35}$-a, c'est expliquer comment les vases pouraient n'oflrir qu'un reflet infidèle du décor originel. Il reste a savoir pourquoi les fabricants - patrons d'entreprises ou petits artisans, sclon la structure que l'on prète hypothétiquement aux officines ${ }^{36}$ - trouvaient leur compte à une production de basse qualité, pourquoi, aussi, marchands et acheteurs s'accommodaient de malfaçons si fréquentes. Il est permis de supposer à ce phénomène différentes causes : penser, par exemple, qu'on procédait à une vente forcée de produits tous aussi mauvais les uns que les autres - - du fait soit de l'incapacité ou de la négligence des ouvriers, soit du rythme abusivement accéléré de la fabrication - ; imaginer, aussi, que la qualité pouvait varier soit entre une vaisselle d'usage courant et des vases fins considérés comme une sorte de pacotille, soit, pour un mème type d'objets, entre des pièces bien venues et d'autres moins belles et moins chères. Cies deux modes d'explication, qui se recoupent partiellement, pourraient être éprouvés si l'on mettait en lumière - ce que je n'ai pu faire dans le cadre d'une brève étude - soit la dégénérescence progressive et générale de la production, soit la coexistence et le synchronisme de produits plus ou moins soignés à l'intérieur d'une série typologique ou, au contraire, selon la forme des vases.

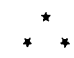

A ne considérer que ces décors à cordons torsadés, il est donc possible de mettre en évidence des groupes structurcls, liés, selon toute apparence, à la fabrication en série et qui répètent, avec des moyens variés, un même schéma décoratif. L'exemple fourni par notre type B 1 , en effet, bien que privilégié, n'est pas unique. Et, sans préjuger de

on the best work of PATEK.VVS, lut the style of decoration, the roped borders, orniments and figure-types were all employed by him, and these moulded vases were undoubtly made in his workship... a 1 propos du vase d'Ilehester "The roped borders and figures are hlurred and the fish is cut by the basal groove. In the style of PATER.VFS, and though it may not have been finished by him it is a product of his workshop."

35 Outre les accidents que perivent provoguer des gestes maladroits at me manipulation precipiter, il faut noter yu'un demoulage premalure destue a accelerer le rylhme de fabricalion, ent raine a lui senl, du fait de l'adherence partielle de l'argile humide à la paroi de la matrice, une delerioration des molifs, dont le modele peut ètre délruit ou brouille.

36 Il nest pas question ici dre meltre en doute l’importance de lobllicine de Paternus pour la produclion des

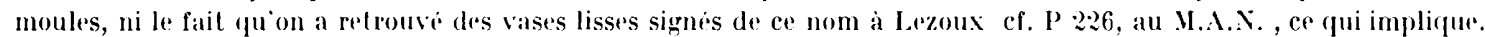
que la fabrication de la vaisselle el celle des matrices put, du moins en principe, ètre groupée a l'intérieur de cetle fabrique. Mais nous ignorons, dans ce cas comme dans d'autres, quelle etait l'organisation et limportance du personnel d'un atelier qui réunissait plusieurs alapes de la fabrication, et si la production de's vases, a lintérieur d’un mème centre, ne pouvait pas êlre partagee entre lentreprise qui creat les moules of des artisans potiers - clients ou sous-traitants du mouliste. lif. pour des exemples dentreprises atlestees daus des centres differents tantot par des moules et des vases lisses signes, tantot seulement par des matrices, B. How wax, les relalions entre potiers, fabricanls de moules el arlistes producleurs de poingons, dans Rei cretariae romanae faulorum Acla XIII, 1973, p. 6-४.. 


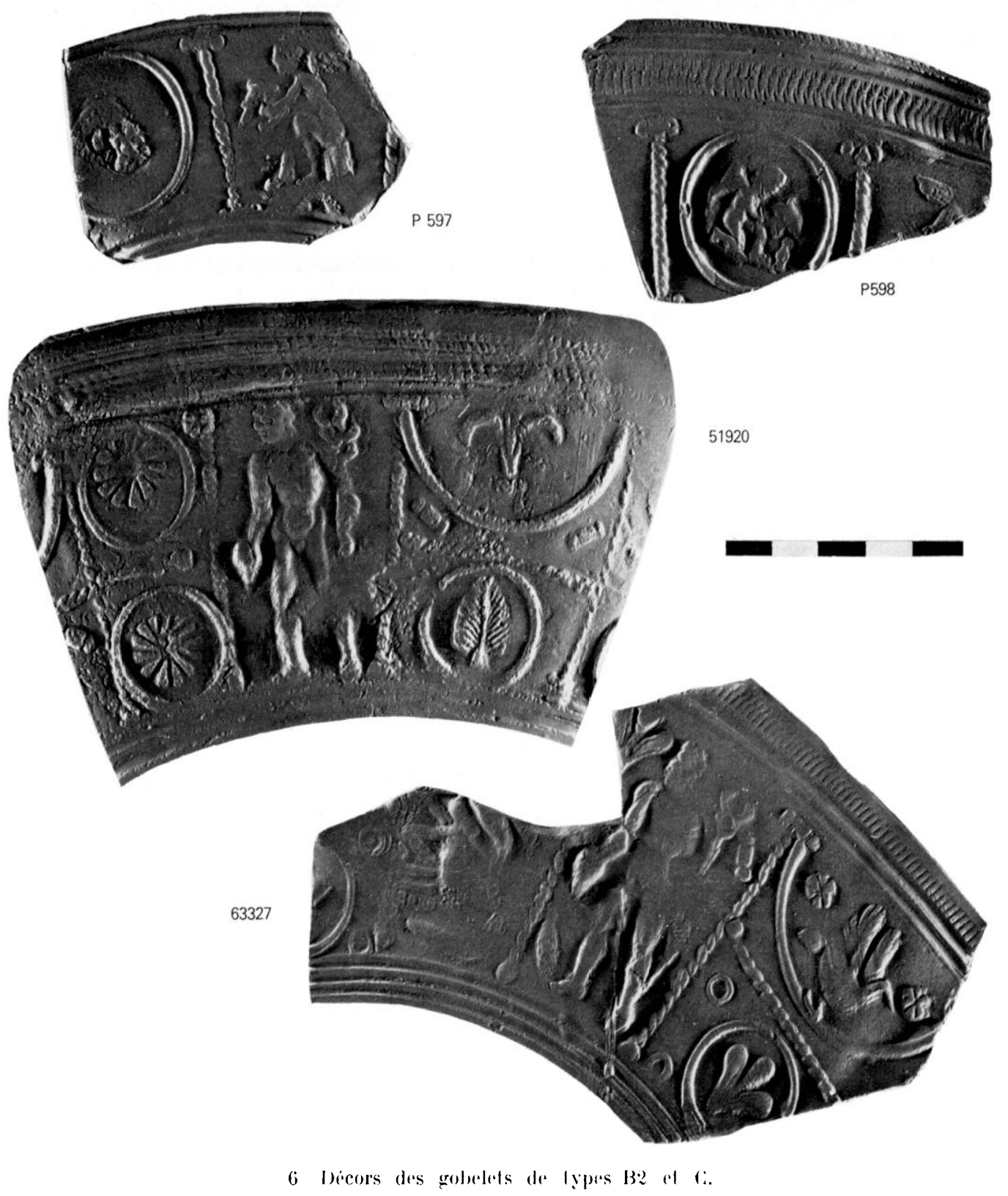

l'importance respective de différents ensembles, qui utilisent le même répertoire, on peut, du moins, signaler l'amore d'autres groupements. Deux autres types de frises sont connus, dans la collection du M.A.X., à deux exemplaires sans parler des fragments isolés, souvent trop petits pour être intelligibles, mais suffisants pour suggérer l'existence de structures différentes de celles que nous analysons ${ }^{37}-$.

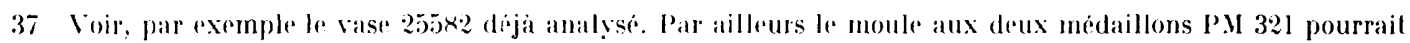
appartenir a une série exclusierment orné d'une frise de cercles habites : ce type de décor est atteste sur au moins

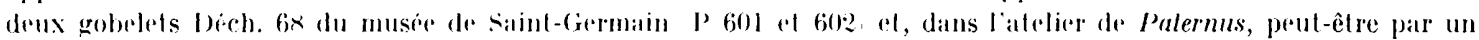

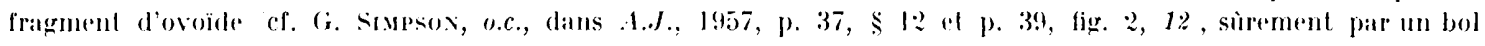

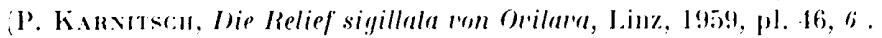




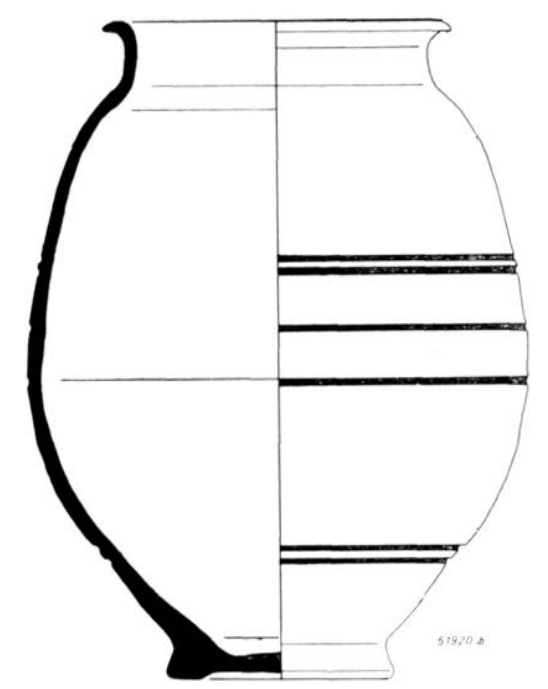

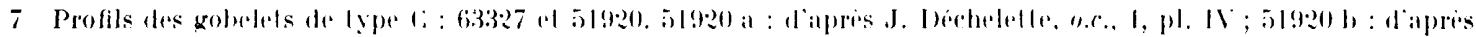
l'original.

Le premier cas est celui de deux petits goledets fig. 4 et 6, P 597 et 598; 8 I3:2). L'un, assez largement conservé, permet de restituer un décor apparemment fonde sur l'alternance d'une métope à sujet libre et d'un panneau à grand médaillon ${ }^{38}$. l'autre, du fait de la similitude des parties conservées - une métope de chaque sorte - et de la reproduction exacte d'une disposition du premier (mème médaillon et cordons de séparation terminés aux deux extrémités par un astragale $R(60)^{39}$, est étroitement lic à celui-ci. La répétition des mèmes poinçons, des mèmes associations et des mèmes constructions sur des vases de taille identique, permet difficilement de croire que les deux échantillons n'appartiennent pas à la mème série.

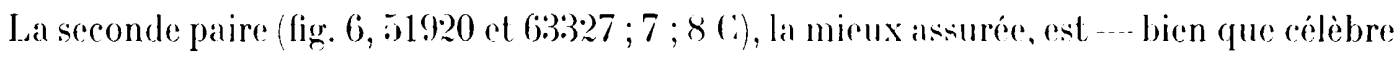
par les références qu'elle fournit depuis 70 ans -. connue de facon approximative. Le premier gobelet, en effet, figure sur une planche de l'ouvage de J. Déchelette ${ }^{40}$; mallheureusement la reproduction du decor et le dessin du profil sont inexarts (fig. 7, 519:20 a et b). Le second fragment, signé, pourrait peut-ètre itre identifié avec le vase signalé dans le catalogue épigraphique de la mème publication. Ce dernier, en tout cals, doit à une description insuffisamment explicite d'avoir été confondu avec l'exemplaire dessinét ${ }^{41}$. L'organisation

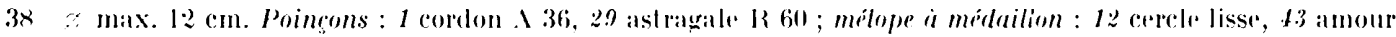

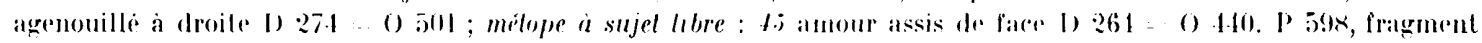
rouge superficiedlement et irreguliemenent bruni.

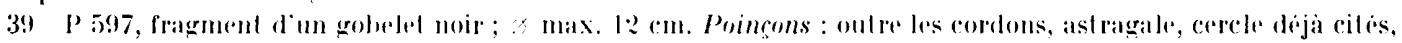

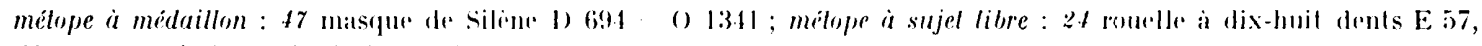
42 amour assis à gauche 1 ) :261 - () 14.4.

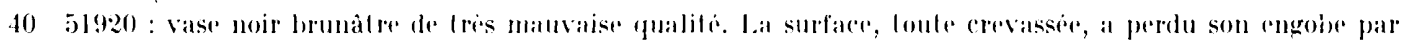

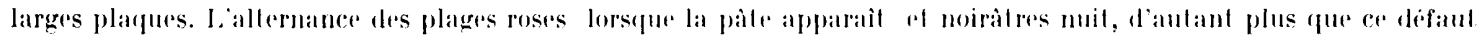

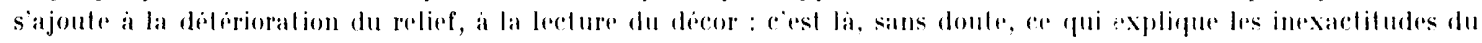

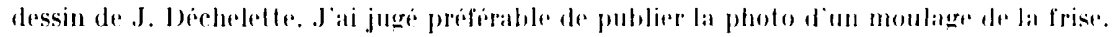

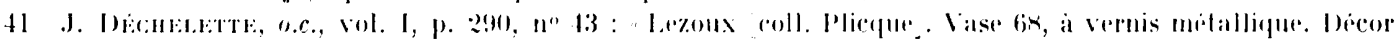

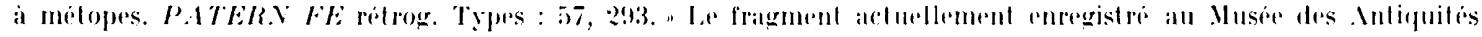

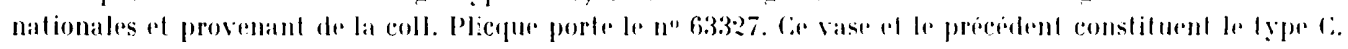


des frises fondée, du fait de la taille des vases, sur des séquences de trois panneaux, comporte, de droite à gauche, l'enchaînement suivant : une métope divisée en deux et contenant, dans le registre supérieur, un arceau habité, dans le registre inférieur un médaillon ; une large métope pleine renfermant un sujet figuré ; un panneau plus étroit, occupé, dans un cas, par un personnage, dans l'autre par deux médaillons séparés par un motif couché. La même suite se répète quatre fois sur le vase largement conservé qu'a reproduit J. Déchelette ${ }^{42}$. Le début d'une deuxième série subsistant à gauche de la première, sur le second gobelet, autorise - compte tenu des habitudes dont témoignent d'autres échantillons - à restituer une composition analogue ${ }^{43}$. Une photographie du moulage de chaque décor et le dessin du profil des deux vases, ainsi que l'inventaire des deux séries de poinçons, permettront de dissiper toute confusion.

51920. $\varnothing \max .15,8 \mathrm{~cm} ;$ haut. $18,7 \mathrm{~cm}$; frise conservée environ aux trois-quarts. Poinçons: 1 cordon A 36,29 astragale $\mathrm{R} 60,16$ rosette à six pétales $\mathrm{C} 123$; métope coupée: 3 arceau lisse ( $\varnothing$ env. $4,5 \mathrm{~cm}$ ) contenant 33 le fleuron trifide $G 56$, même astragale, 11 petit cercle lisse $(\varnothing$ env. $2,5 \mathrm{~cm})$ contenant 35 la palme J 119 ; large métope pleine: 39 Mercure D $289=0529$; mélope pleine étroite: même petit cercle lisse contenant 22 une grande rosette à treize pétales obliques; 28 colonnetle sur une base P 68 . Vase publié par J. Déchelette.

63327. " max. $16 \mathrm{~cm}$; fragment d'un vase signé composé de deux morceaux et couvrant environ un quart de la frise ${ }^{44}$. Poinçons: 1 cordon A 36,29 astragale R 60, 32 fleuron G 153,15 pois ; métope coupée: 3 arceau lisse (cf. 51920) contenant 16 des rosettes à six pétales C. 123 et 49 un oiseau aux ailes éployées à droite I) $1010=02316,11$ petit cercle lisse (cf. 51920) contenant 32 un fleuron trifide $G 153,8$ petit anneau $(z 0,5 \mathrm{~cm})$; large mélope pleine: 39 Mercure D $289=0529$, même astragale, 26 tortillon fusiforme ; mélope pleine étroite: 40 Apollon assis vers la droite D $57=094$, tortillon fusiforme ${ }^{45}$, estampille [pate]R $\widehat{\mathrm{FE}}$.

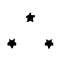

L'examen de vingt-neuf échantillons, quatorze moules et quinze vases, conduit à définir un ensemble homogène de différents points de vue : la typologie est cohérente, la morphologie des moules uniforme ${ }^{46}$; le module des gobelets achevés, comme celui des exemplaires supposés à partir des matrices, varie peu pour une même catégorie de frise;

42 In fragment passé inaperçu au moment de la première restauration et que j’ai retrouvé récemment donne la certitude formelle que le llercure se répétait uniformément; les quatre séquences géométriques identiques étaient dejà toutes au moins partiollement attestées.

43 Sur l'organisation des décors en séquences orientées dans le mème sens, à lexoux à partir du règne d'Hadrien, cf. 1 propos de Calussa, p. 100-107. On ne saurait préjuger de la répétilion uniforme sur ce gobelet de tous les poincons, ou de l'usage de motifs alternés. Tout au plus peut-on estimer, compte tenu de l'emploi fréquent des oiseaux par couples cf. supra, pour cette série, 32251 et 32412 , que l'alternance d’un oiseau à droite avec un oiseau à gauche n'a rien d'invraisemblable.

44 Cif. infra, Appendice, p. 197.

45 Il s'agit probablement dans l'une et l'autre métopes du même poinçon imprimé plus ou moins complètement. les deux motifs s'enroulent dans le même sens et doivent s'identifier avec l'un des lortillons de Paternus II (ce. S.S., fig. 30,18 .

46 On ne saurait cependant déduire de cette constance - observée sur des malrices dont le diamètre intérieur varie entre 9,5 et env. $15 \mathrm{~cm}$, que tous les moules destines au même usage dans le même atelier avaient nécessairement la même forme : on conslate une certaine diversité de forme dans ces séries de matrices et différents facteurs peuvent, hỵpothétiquement, en déterminer les variations, la date, le goùt du fabricant, la forme ou le module du vase prévil. 
la structure des décors rend une vingtaine de pièces comparables entre elles. Le répertoire, enfin, établit un lien solide à l'intérieur du groupe : en effet, les mêmes poinçons se retrouvent dans des associations varićes et les exemples uniques ${ }^{47}$ sont toujours isolés au milieu d'une série de motifs mieux attestés, si bien que cette particularité ne saurait suffire à exclure tel gobelet, ou tel autre, de cette famille. Les relations à l'intérieur du groupe sont mises en évidence dans deux tableaux : l'un (p. 188) présente décor par décor les combinaisons de motifs et montre, en même temps la communauté de répertoire d'une pièce à l'autre, soit à l'intérieur d'un mème type de frise, soit entre deux types; le second (p. 200-202) fournit, avec la liste des poinçons, l'inventaire des motifs associés à chacun d'eux sur nos moules et vases.

Par ailleurs, le recensement, provisoire, du répertoire de ces gobelets permet de préciser les rapports du matériel iconographique ici utilisé avec celui du potier Paternus $I I$ : c'est le nom de ce fabricant qui figure sur le seul exemplaire signé ; c'est à son atelier qu'on a attribué les trois gobelets déjà publiés de notre série ${ }^{48}$. Pour les motifs non figurés, je me suis référée au catalogue de ( $\mathrm{x}$. B. Rogers que j'ai complété principalement à l'aide d'inédits (fig. 9). L'inventaire des poinçons comprend deux catégories de motifs. Les premiers ${ }^{49}$, purement géométriques et sans particularités notables (cercles et arceaux lisses), ou difficilement caractérisés (tortillons fusiformes) (fig. 9, 2-5, 8-15, 26), ont été laissés de côté par G. I3. Rogers, parce que leurs dimensions pouvaient varier considérablement selon la qualité de l'impression ou la rétraction de la pâte. Dans la mesure où ils n'étaient effectivement affectés d'aucune caractéristique, j'ai pu les identifier lorsque j'avais entre les mains des moules ou des vases, signés ou d'attribution peu douteuse, qui confirmaient l'emploi, dans l'officine de Palernus $I I$, d'un poinçon de même aspect et de mêmes dimensions (cf. fig. 10,4), mais il a été généralement impossible de recourir aux dessins de tessons fournis par les publications : les projections planes déforment une partie de ces décors; de plus, la reproduction des motifs strictement géométriques est, par elle-même, sujette à de fréquentes variations. Il est donc probable que les attributions que j'ai faites ne représentent qu'un minimum et que l'inventaire direct d'un matériel plus abondant permettrait de combler certaines lacunes - sans, pour autant, donner nécessairement des preuves de la spécificité de l'emploi de la plupart de ces éléments de décor -... Ces derniers, en fait, ne semblent devoir jouer que le rôle d'un appoint dans le travail d'identification. Parmi les poinçons proches de cette catégorie, mais individualisés, on relèvera le double arceau intérieurement strié -- absent de l'inventaire de G. B. Rogers, il ne m'est pas autrement connu - et le double arceau outrepassé (fig. 9, 7 et 6 ). Cie dernier, également absent du catalogue, est associé sur un autre fragment de gobelet de Saint-Germain au cordon torsadé, au coq et surtout à la palme, attestée, jusqu'à présent, avec la seule signature de Paternus $I I^{50}$. Ajoutons que ce motif est combiné, sur nos vases,

47 Jos 4,10,13,14,20,34, 40 du calalogur. D'autres motifs, qui figurent une seule fois sur nos moules el gobelets, sont ailleurs attestés et altribués à Palernus.

48 II.A.X. 519:20, dans S.S., pl. 104, 1 et les vases d'Ilchester et de I.incoln.

49 Nos 2-1; el 26 du catalogue.

50 Vase P 599 




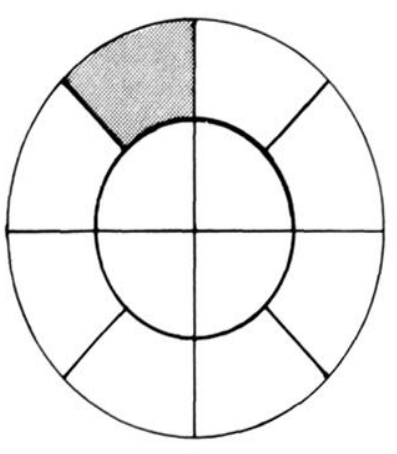

A

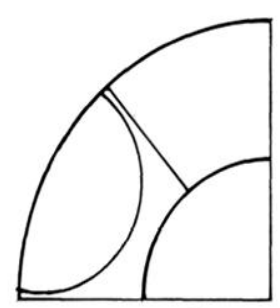

B 1

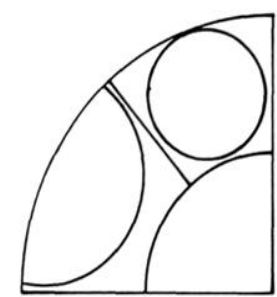

B 1 a
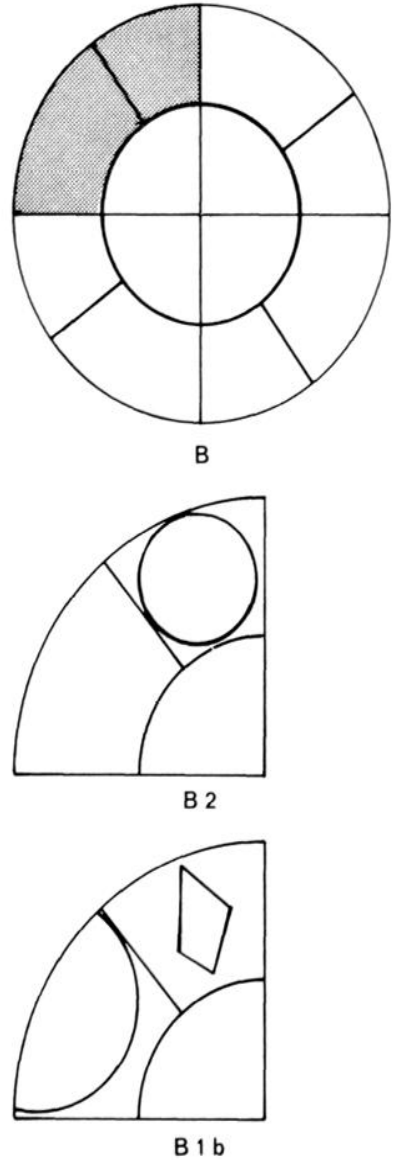
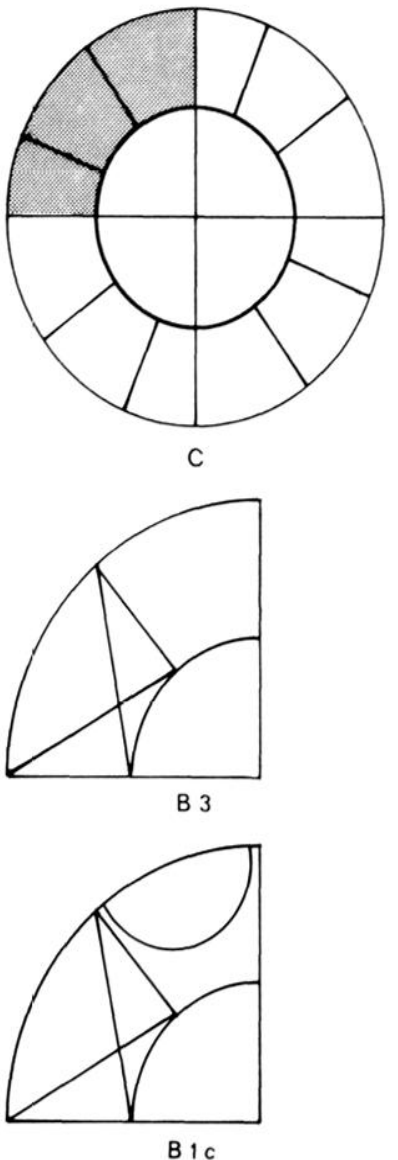

8 Schémas des types, séries et sous-groupes de décor. En gris : l'élément de base de chaque découpage.

avec plusieurs poinçons $(1,8,24,29,33$ de ma liste) qu'on ne trouve conjointement employés que sous la signature de Palernus.

Parmi les vingt-deux autres motifs secondaires (fig. 9, 1, 16-25, 27-37), seize ont été retenus sûrement, ou probablement par G. B. Rogers : dix sont attestés avec la signature de Palernus ${ }^{51}$, dont un - la palme - avec cette seule estampille; quatre sont attribués au style de Palernus dont deux de façon exclusive ${ }^{52}$; le cinquième et le sixième n'étaient encore signalés que dans d'autres ateliers ${ }^{53}$. Parmi les six derniers décors, deux ont résisté à l'enquête ${ }^{54}$ et apparaissent comme des nouveautés, deux ont été identifiés d'après des publications $\mathrm{s}^{55}$; un autre est maintenant connu sur un tesson signé ${ }^{56}$ (fig. 10,1 et 3 ). Le dernier figure sur un fragment de gobelet attribuable à Palernus II ou à quelqu'un de

51 I.es nos $1,16,19,23,24,28,29,32,33$ et $3 ;$ du catalogue.

52 Les nos $20,30,36$ et 37 . On ne connaìt que cette altribution pour 30 et 36 .

53 Les $n^{\circ s} 31$, le fleuron bifide, el 34 , la feuille à cinq pointes. Le premier, attesté dans le style de Secundinus $I I I$ et celui de Sissus $I I$, s’ajoute à la liste des poinçons communs à Sissus et Palernus. L.e second n'était encore attribué qu'au style d'Albucius.

54 Les nos 18 et 21 , deux roselles.

55 Les $n^{\text {os }} 17$ (S.S., fig. 30, 3 : Paternus; et 27 (S.S., pl. 120, 6 : style d'Albucius; peut-être pl. 105, ; : Palernus $I I$.

56 Ie $n^{\circ} 22$, une grande rosette à treize pétales atlestée dans la coll. Mathonnière-Plicque du musée de Cannes. 

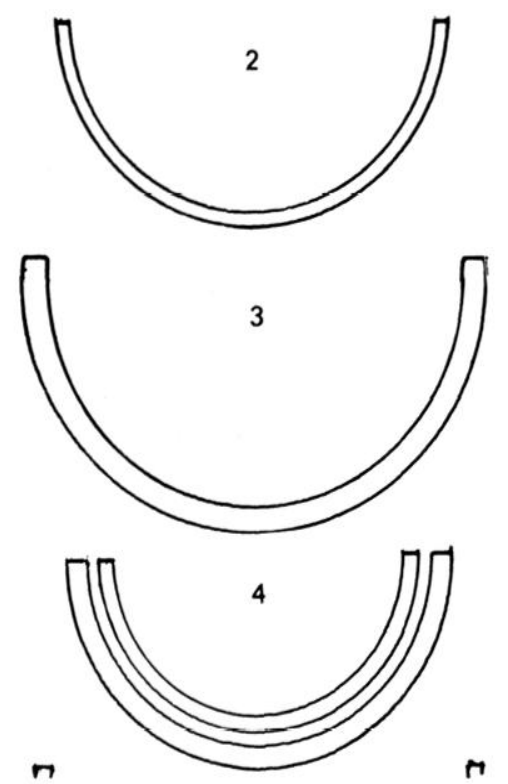

की

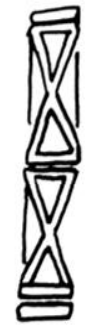

20 E



2

19

21

22

23

27

2

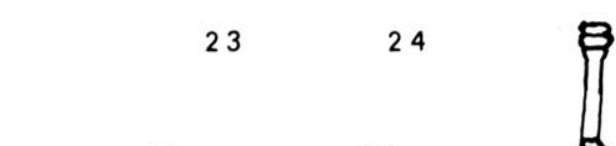

200000000 00000000

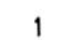

25

26
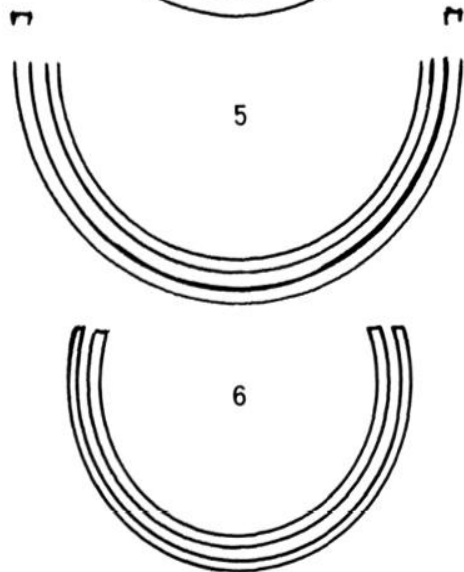

得

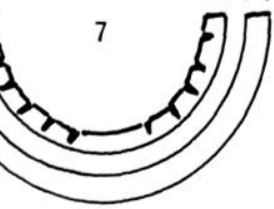

(1)

8

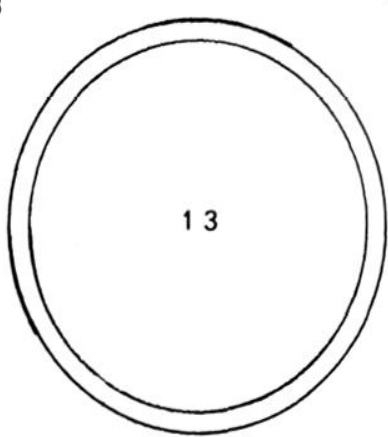

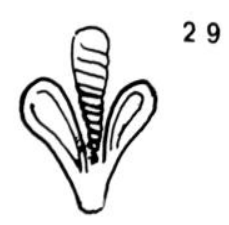

32

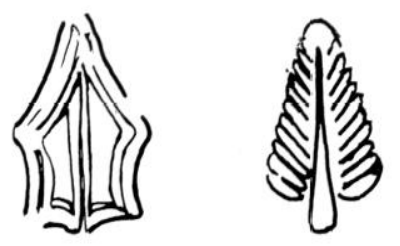

34
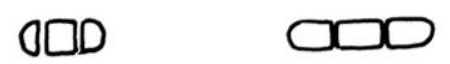

28

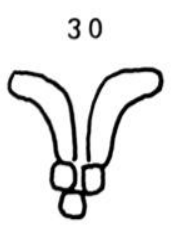

31

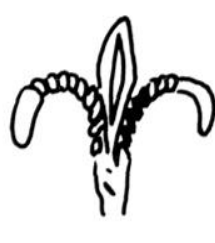

33

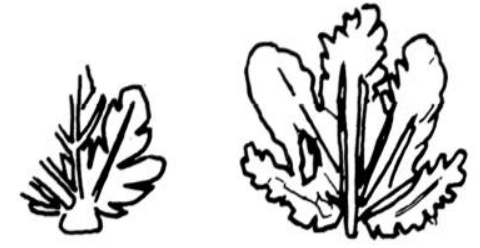

37

36

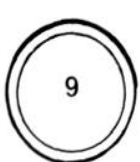

$3 \mathrm{~cm}$
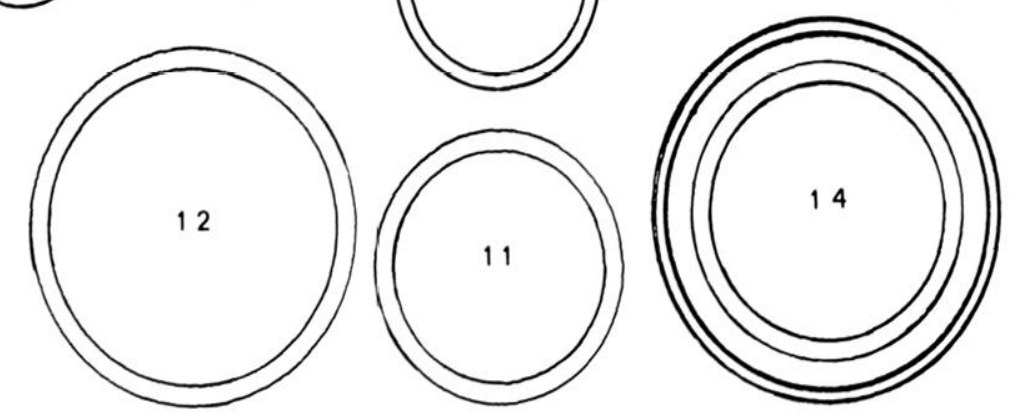

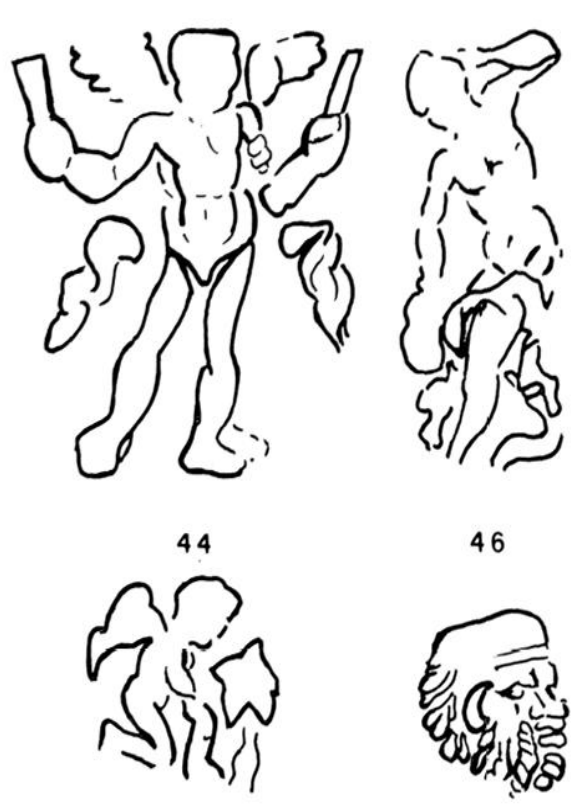

46
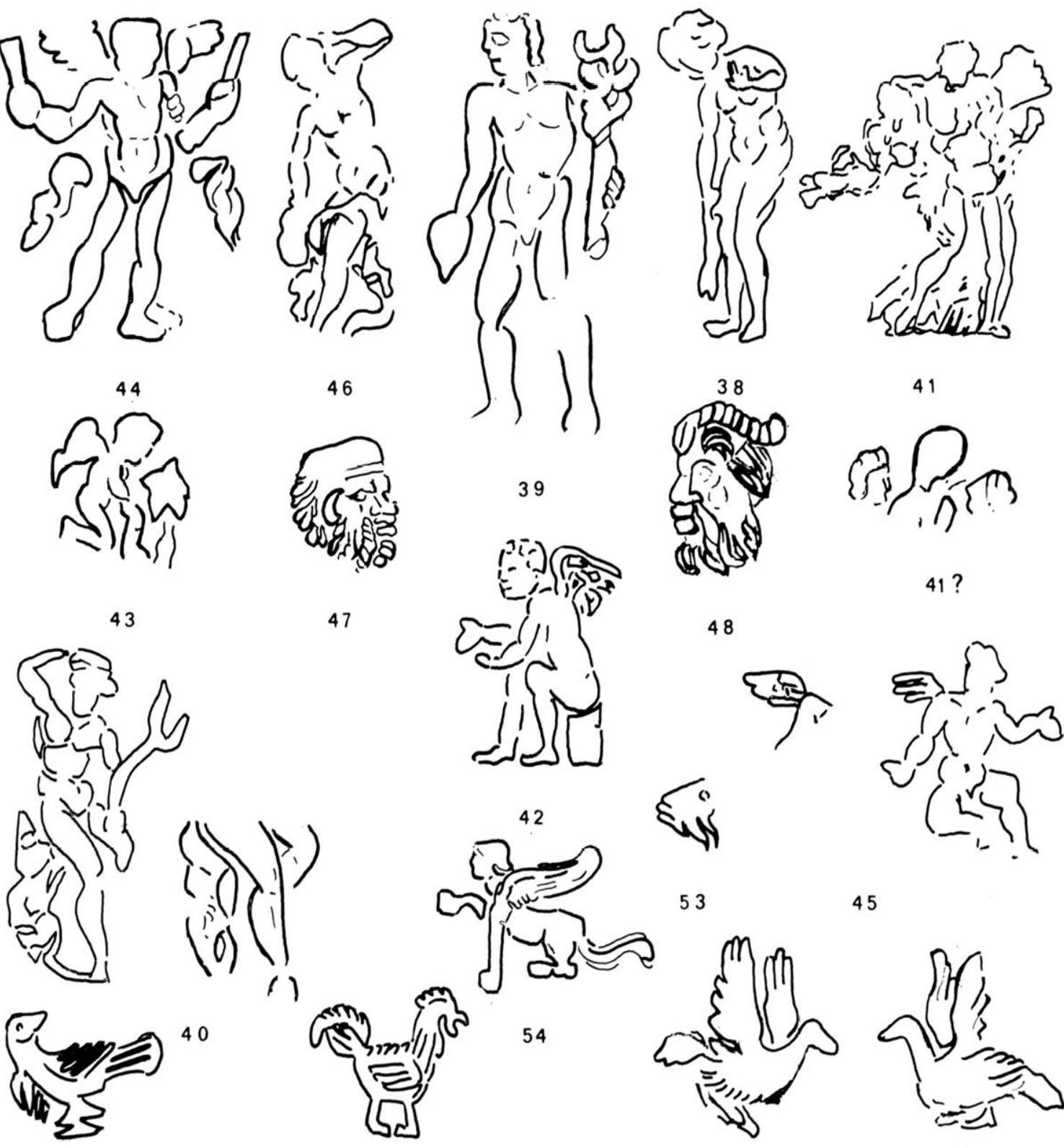

47
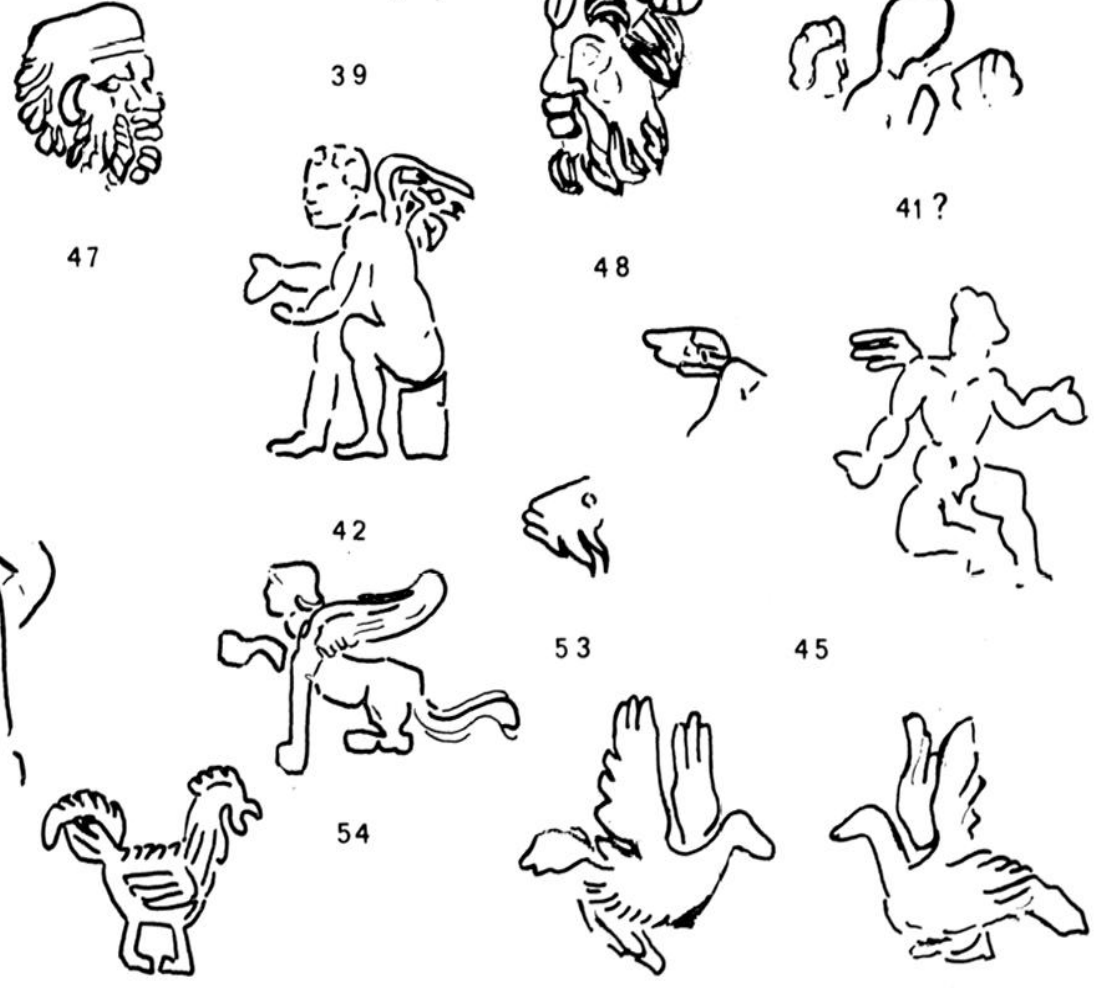

51

52

49

50

9 Catalogue des poinçons décoratifs : le groupe bacchique 41 reproduit le dessin du vase de Lincoln, la roselte 20 a la mème origine; l'Apollon et l'amour 45 entiers figurent sur des bols D. 37 signés conservés au M.A.X. (respectivement $66491(A 7$, et 66111$)$. 




10 Poinçons isolés ou associés altestés sur des bols D. 37 signés. 1 et 3 : fragments de Cannes (coll. Mathonnière-Plicque ; 2 (66491 (B1) et 4 (66491 (A1)) : M.A.N. (coll. Plicque).

son entourage ${ }^{57}$. L'inventaire permet donc d'ajouter huit poincons au catalogue de G. B. Rogers et deux attestations nouvelles de décors connus ${ }^{58}$.

Le recensement des motifs figurés (fig. 9,38 et suiv.) suscite deux remarques : ils sont relativement peu nombreux -- dix-sept sur cinquante-quatre - et, pour la moitié environ, de petite taille. L'un et l'autre de ces phénomènes s'expliquent principalement par les dimensions des vases : un seul des poinçons, le Mercure, frôle les $6 \mathrm{~cm}$, les plus hautes des autres figures atteignent environ $4,5 \mathrm{~cm}^{59}$. Mercure ne se trouve que sur les plus grands des gobelets ${ }^{60}$, la plupart des autres, sur des pièces de la taille la plus courante ${ }^{61}$. Les contraintes imposées par la hauteur de la frise, qui varie avec le diamètre, paraissent, en la circonstance, avoir réduit le nombre des motifs figurés utilisables : la similitude des poinçons figurant sur les gobelets et sur des grandes formes classiques, tels les bols, laisse à croire qu'on employait, dans la mesure du possible, le même outillage pour tous les types de vases ${ }^{62}$.

Tous les motifs figurés sont déjà connus ${ }^{63}$. Cependant, l'un d'eux, le groupe bacchique

57 Le $\mathrm{n}^{\circ} 2.5$. Cette torsade rectiligne figure sur un gobelet noir, surcuit (P 600), de la coll. Plicque portant un décor de type B 2. Poinçons : cordon perlé $\Lambda 2$, amour assis à gauche D $261=0$ ) 444, cercle lisse (cf. 12), oiseau à gauche $\mathrm{D} 1011=02324$ ?

58 Les $\mathrm{n}^{\text {os }} 6,7,17,18,21,22,25,27$ et, d'autre part, 31 et 34 . J'ai attribué aux premiers, avec l'accord de G. B. Rogers, des numéros disponibles dans son catalogue : F 63, F 62, C 137, E 66, E 67, C 240, P 27, P 7.

59 Vénus, Apollon, groupe bacchique, amour debout, personnage assis.

$60 \quad 51920$ et 63327.

$61 \varnothing$ max. env. $12 \mathrm{~cm}$.

62 De même l'absence de certains poinçons très allongés et adaptés à la faible courbure des grands bols (par exemple les grands dauphins) peut trouver son explication dans des raisons techniques (courbure des petits moules) autant qu'esthétiques (disproportion des motifs;.

63 c. tableau II. 
(fig. 9, 41) semble offrir une variante du décor recensé et illustré par J. Déchelette ${ }^{64}$. Dix sont attestés avec la signature de Paternus II par F. Oswald, J. Déchelette ou J. A. Stanfield et $\mathrm{G}$. Simpson ${ }^{65}$ et un vase signé du Iusée des Antiquités nationales permet d'en ajouter un onzième ${ }^{66}$ (fig. 10,2). Quatre autres motifs sont déjà considérés comme appartenant au style de Palernus ${ }^{67}$, mais je n'ai pas encore rencontré les deux derniers - un masque et un personnage assis ${ }^{68} \ldots$ sur des pièces sùrement attribuables à ce potier. La tête de Silène est un héritage de l'époque de Liberlus, dont on n'a pas suivi le développement. En revanche, la permanence, ou la résurgence, du poinçon représentant un personnage assis, un bras levé et plić vers la tête, connu par des vases d'Igocalus $(X-4)$ et d'autres dans le style d'Aduocisus ${ }^{69}$. est garantie dans l'entourage de Paternus par un moule D. 37 proche, par son répertoire, à la fois des produits de notre potier et de ceux d'Aduocisus ${ }^{70}$ (fig. 11, PM 360). L'identification de ces décors ne posait pas de problème et n'apportait pas d'inédits, je me suis donc attachée (cf. tableau II) à rassembler des preuves que le même poinçon était utilisé par Palernus II ou, dans le cas de sujets comportant de nombreuses variantes (cf. fig. 11, P.I 354), à différencier l'exemplaire figurant sur nos vases des modèles légèrement dissemblables assortis d'autres estampilles ${ }^{71}$.

Pour la structure des frises, l'alternance de deux ou plusieurs panneaux, coupés ou pleins et s'enchaînant uniformément dans le même ordre, et l'emploi d'arceaux et de médaillons, constituent des caractères tellement fréquents des vases $d_{\mathrm{u}} \mathrm{II}^{\mathrm{e}}$ siècle, à Lezoux, qu'il convient à peine de les rappeler. Tout au plus doit-on signaler que la sécheresse des décors, en particulier dans le choix des motifs de séparation et de remplissage, est tout à fait conforme aux usages de la seconde partie de ce siècle ${ }^{72}$. Si l'on veut isoler des particularités plus spécifiques, on doit les chercher plutòt dans le détail de certaines organisations. On constatera ainsi que l'écartèlement de panneaux en sautoir, sous une forme nouvelle, est attesté autour de P'alernus $I I$ ou chez ses prédécesseurs directs. En effet, alors que ce type d'ornement, à la fin du ier siècle à la Graufesenque et au début $\mathrm{du}{ }_{\mathrm{II}^{\mathrm{e}}} \mathrm{a}$ Lezoux se présente comme une composition de petits éléments géométriques

64 Cf. (G. Simpsox, o. c., dans A.J., 1973, p. 50.

65 Les $n^{\circ} 38,39,40,42,44,4 j, 48,49,50,54$ du catalogue.

666649 ( (B 1). La preuve est solide, mais indirecte. I'oiseau stylisé à gauche (51, fait partie d'un couple constitué d'un oiseau a gauche et d'un autre à droite qui, comme d'autres paires cf. v 1010 et 1011 , sont d'ordinaire attestés ensemble. Le fragment du M.A.X. conserve, dans un rinceau, l'oiseau à droite, associé à la signature PATEIRN FE.

67 Les nos 41 : groupe bacchique (G. Simpsox, l.c. ', 4.3: amour à genoux (S.S., pl. 109, 3), 52: coq (P). KarNitsch, o. c., pl. 55, j), 53 : poisson (G. Simpsox, l.c. .

68 Les $\mathrm{n}^{\mathrm{os}} 47$ et 46.

69 S.S., pl. 18, 23.3 pt pl. 114, 28.

70 PM 360. Poinçons de Palernus $I /$ : cordon I 36, pois, astragale $\mathrm{K}$ 60. Houble cercle a lorsade extérieure E 10 'cf. l'exemplaire signé inédit M.A.X. 66110 , rosette à douze pétales (style de P... Poincons d'Aduocisus : Apollon D $45=077$, femme drapé à droite I) $505=0$ 929. L'ove B 137 qui borde ce décor n'est encore attribué ni à l'un ni à l'autre.

71 Ainsi sonl garanlis Mercure, l'oiseau à droite Gobelet 63327), Apollon (gobelet et bol 66491 A 7 , l'amour assis à gauche (bol 66491 i 13 ) l'amour assis de face bol 66491 (A 17 )), l'amour debout (bol 66491 (B 7 )!, l'oiseau à gauche (bol 14511), le sphinx (bol 256.13). Le masque cornu differe des exemplaires signés de Quintilianus, Sissus 11 , Calussa $I$ et je ne l'ai pour le moment retrouvé que sur des échantillons par ailleurs at tribuables à Paternus. I.e groupe bacchique et la Vénus sont différents, ('n tout cas, des poinçons de Libertus, le coq, de celui de Calussa I.

72 C.f. 1 propos de Calussa, p. $98-102$ et 110. 


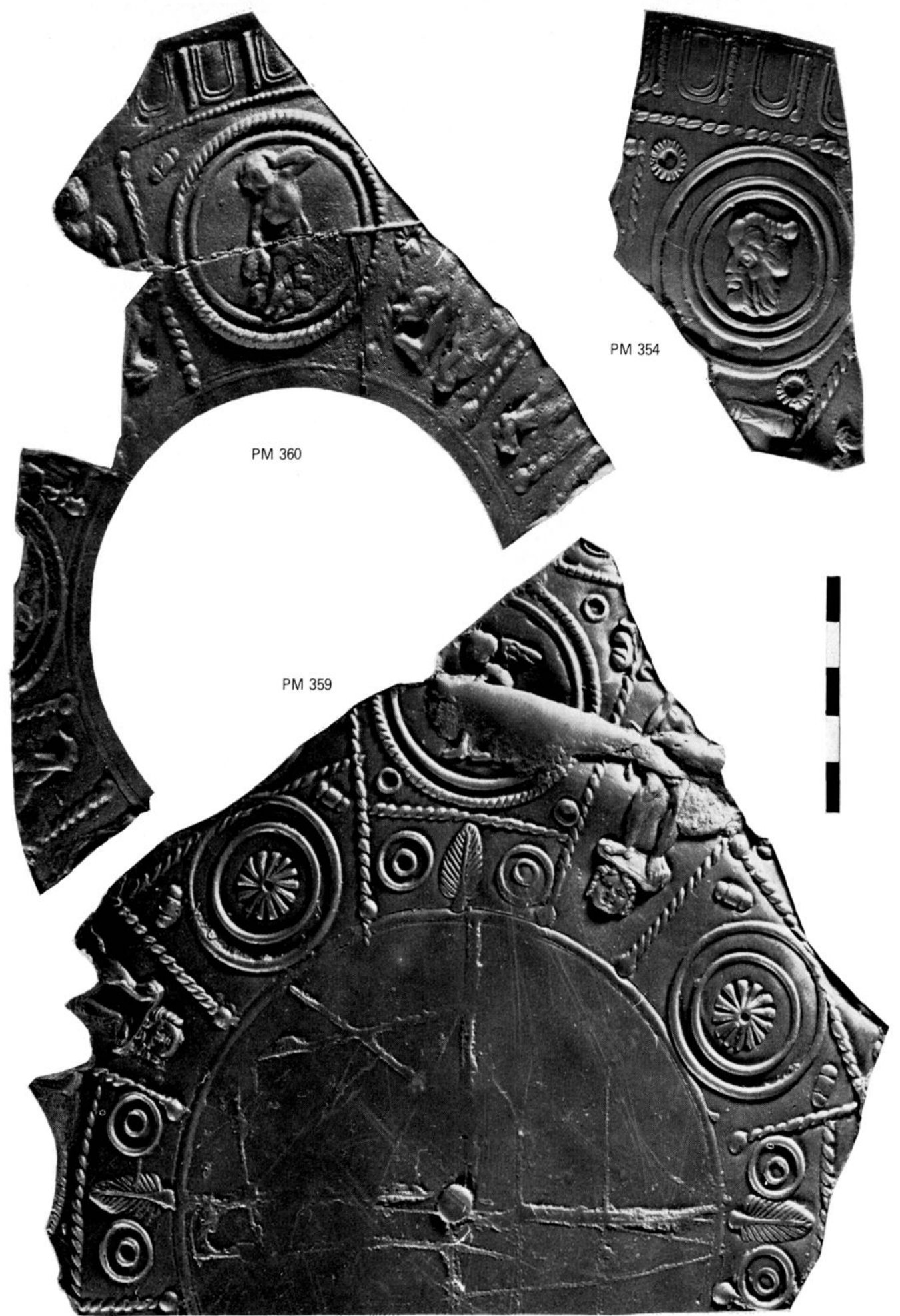

11 Poinçons et motifs composites atlestes sur des moules D) 37 (M.A.X., coll. Plicrue. P.11 354 el 359 : style de PATERNVS II (masque cornu bien conservé ; poinçons divers el construction à symétrie bilatérale; ; P.l 360 : style de? (personnage assis). 
ou végétaux, rayonnant autour d'un centre, auquel ils sont rattachés ${ }^{73}$, l'habitude se crée, timidement avec Auitus-Vegetus ${ }^{74}$, plus nettement avec Quintilianus ou ses associés ${ }^{75}$, de composer des métopes dont les motifs - géométriques, végétaux ou figurés - emplissent chaque quartier en respectant certaines règles de symétrie, mais sans être aucunement reliés au centre du sautoir. Ce type de structure, que l'on rencontre sur deux de nos vases ${ }^{76}$, est attesté - avec rles poinçons végétaux, géométriques et un oiseau - - sur un moule Déch. 68 de Palernus $I^{n}$, - avec un mélange de grandes feuilles et de petits motifs végétaux - sur des formes classiques ou des gobelets dus à Censorinus et Laxtucissa ${ }^{78}$, - avec des décors géométriques ou des végétaux stylisés -- sur des I). 37 de Iustus et de Calelus-Seuerus ${ }^{79}$, - avec des sujets végétaux et animaux -- sur quelques bols de Cellus $^{80}$. Le répertoire des deux vases ovoïdes de notre série s'apparente, par la nature des poinçons (du moins d'après ce qui reste), aux motifs géométriques ou aux végétaux stylisés et sans grâce de Iuslus, mais le vase le mieux conservé, celui de Vichy, présente une composition plus riche que celle des bols de Iuslus, si on juge, du moins, d'après les exemples publiés dans Central Gaulish Pollers.

On peut montrer, de la même façon, que les motifs à symétrie bilatérale, composés de trois éléments, se retrouvent sur des gobelets de Laxlucissa ${ }^{81}$, et sur des bols soit de l'atelier de Palernus ${ }^{82}$ (cf. fig. 11, P.I 3599), soit de potiers contemporains ${ }^{83}$. Selon la hauteur de la frise, le registre supérieur est occupé tantôt par des arceaux, comme sur nos gobelets, tantôt par des médaillons.

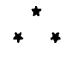

L'analyse de cette série d'objets a permis d'attirer l'attention sur des questions pour une part habituelles et lièes à l'attribution d'un ensemble presque entièrement anonyme, pour une autre déterminées par la composition particulière du lot étudié : le groupe, comprenant des instruments de fabrication et des vases finis, invite à s'interroger sur les

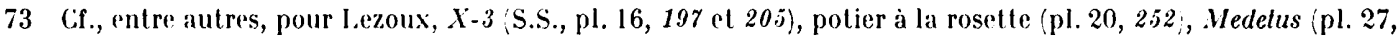
324, Medelus-Ranlo (pl. 29, 25.3) Inenalis (pl. 35, 413), Donnaucus (s) (pl. 43, 493).

74 Ces compositions, qui ne sont pas exclusives des sautoirs traditionnels, combinent des décors rayonnants (géométriques ou végélaux) et des motifs animaux remplissant le champ (S.S., pl. 62,$3 ; 63,11$ ct $13 ; 64,22$ ). Le même genre de mélange se retrouve sur certains produits du groupe de Quintilianus (pl. 70, 18).

75 o.c., pl. 71, 29, bol auquel on peut ajouter un moule de gobelet tronconique (P.I 262 orné de feuilles, de rosettes, d'une lyre et d'un fleuron bifide.

7625582 et P 593.

77 P.I 293.

78 Censorinus : bol (S.S., pl. 103, 19); Laxlucissa (s) : moule d'ovoïde P.I 235.

79 Iustus (S.S., pl. $110,7 \mathrm{et} 8$; Calelus-Seuerus (ibid., pl. 128, 8).

80) S.S., pl. 141, 8 el 142, 29 ; P. KarNitscit, o.c., pl. 80, 1.

81 Cf. un moule de gobelet signé de Laxlucissa 32421 , qui porte, de part et d'autre de la colonne sur un socle, un petit fleuron et une tortue, et un vase attribuable au même atelier (P 603) qui présente, sous l'arceau un motif composé de deux doubles-cereles, renfermant chacun une rosette à sept pétales, et d'un large fleuron trifide (S.S., fig. 27,2 .

82 (if. un bol signé (14511) : un motif composé de deux fleurons bifides ( 371 et d'un tortillon fusiforme; un second (P. Karvitsch, o.c., pl. 48,2): motif composé d'un arbre el de deux fleurons, sans compter plusieurs moules et vases dans le style de Paternus.

83 Iuslus (ou Paternus ?) : S.S., pl. 111, 18; Aduocisus (s) : pl. 113, 26; Priscus (s) : pl. 129, 4 ?. 
relations entre moules et gobelets ; les décors, nombreux et remarquablement homogènes, révèlent l'existence de schémas mécaniquement répétés et dont il est possible d'établir ou de pressentir le classement; enfin, l'abondance relative des témoins imputables à un même atelier, le bon état de plusieurs d'entre eux et certains traits, moins banals que d'autres, de l'organisation des frises donnent le moyen de situer stylistiquement ces compositions, par référence à la vaisselle classique, que représentent les bols, ct par rapport au répertoire et aux structures des décors en usage dans quelques ateliers de Lezoux, au II $^{\mathbf{e}}$ siècle.

L'examen du matériel, avant toutes choses, donne le moyen de concevoir avec une plus grande vraisemblance les conditions de la fabrication; en particulier, la grande différence de qualité entre vases et matrices fournit un argument en faveur de l'hypothèse du partage du travail et de la spécialisation des ouvriers. D'autre part, la constance du module des gobelets et des moules, la régularité morphologique de ces derniers, l'influence apparente de la taille du récipient sur la répartition de la frise en un nombre plus ou moins grand de panneaux, la remarquable monotonic du schéma d'organisation d'un nombre important de décors, toutes ces particularités permettent d'avoir une idée plus précise des méthodes de fabrication. Enfin, la qualité défectueuse de nombreux produits finis pose un problème dont la solution demeure incertaine : s'agit-il d'une dégénérescence générale, liée à la date ou aux conditions de production, ou d'un mépris sélectif de la perfection attaché exclusivement à certaines catégories de vases? Nous ne pouvons actuellement le dire.

Par ailleurs, l'ensemble des décors apporte une connaissance nouvelle d'un répertoire apparemment homogène, si l'on considère les combinaisons multiples des différents poinçons entre eux : nous découvrons ainsi soit des motifs peu connus soit une utilisation inédite de poinçons autrement attestés. On peut -... d'autres l'ont déjà fait et notre inventaire ne va pas formellement à l'encontre de cette approximation - attribuer cet outillage à Paternus $I I$ : c'est sous son nom, en effet, que l'on place l'association du plus grand nombre des poinçons. Toutefois, on peut aussi être frappé du nombre des éléments de décor non répertoriés encore avec la signature du potier et réserver la possibilité que cette fabrication, actuellement distincte de la plupart des vases déjà publiés, conserve des caractères particuliers : elle pourrait, par exemple, être imputable à un moment de l'activité de Palernus ou à un décorateur associé. Seule la mise au jour d'échantillons plus nombreux et, de préférence, signés permettra de confirmer l'une ou l'autre de ces hypothèses. En tout cas, l'étude de structures de détail confirme l'attribution et la datation globale de ce matériel : certains types de construction sont utilisés principalement par des potiers qui partagent avec Paternus $I I$ une partie de son répertoire décoratif et à une époque où l'on place l'essentiel de la production de cet atelier, soit entre environ 160 et au moins 190 .

Enfin, l'analyse comparée des décors de bols et de gobelets met en évidence les solutions choisies pour résoudre, sur ces petits vases fins, le problème posé par la réduction de la surface à orner. Elles paraissent consister en une adaptation aux moindres frais d'un répertoire et de structures couramment utilisés pour des formes classiques souvent plus grandes. L'accommodation de l'outillage se fait par sélection : sont éliminés, quoi qu'ils 
représentent, les poinçons incompatibles avec la hauteur, ou la longueur et le galbe de la frise. Ce qui explique la prolifération des motifs secondaires ou des sujets figurés de petite taille. Il n'est jamais apparu, en effet, que l'on utilisât, pour ces vases, des répliques, réduites à cette seule fin, de grands sujets. A-t-on créé, en même temps, de nouveaux poinçons, propres aux décors de gobelets? ou employé des motifs déjà connus, mais réservés, dans cet atelier, à ce type de production? Cette hypothèse, parmi d'autres, expliquerait le nombre des inédits, partiels ou totaux, que nous avons signalés, mais elle n'aurait de fondements que si notre connaissance de la production de Palernus II était moins partielle et sommaire. Quant aux principes d'organisation des panneaux, ils sont en tous points comparables à ceux qu'on observe sur des formes classiques et l'on procède par simplification et élimination : réduction du nombre des métopes quand la longueur de la frise et la taille moyenne des motifs le commandent, mais maintien du nombre habituel des séquences de panneaux ; adaptation du mobilier décoratif à un découpage en registres resté semblable.

Ainsi, malgré les questions encore en suspens, le regroupement d'un ensemble apparemment dépourvu d'attraits, conduit à une connaissance plus large d'un répertoire homogène et fournit l'occasion d'envisager de façon concrète deux problèmes : celui souvent négligé - des relations entre les gobelets et les gros vases moulés, celui des modalités d'une production qualifiée, souvent sans justification très explicite, d'industrielle.

APPENDice

L'identification du vase 63327 avec le $\mathrm{n}^{0} 43$ du catalogue épigraphique de J. Déchelette se heurte aux difficultés suivantes: Déchelette signale un vase 68 noir, métallescent de la coll. Plicque, portant la signature PATERN FE et, dans un décor à métopes, l'Apollon 57 et le Mercure 293 de son inventaire des poinçons. Le vase est le seul qu'il connaisse de cette espèce dans une collection qui, selon son témoignage, a été entièrement acquise par le musée des Antiquités nationales et qu'il a étudiée d'abord à Lezoux, puis à Saint-Germain ${ }^{84}$. Le matériel actuellement conservé au M.A.N. contient un fragment de gobelet Déch. 68 noir, métallescent, composé de deux morceaux. L'un, numéroté $63327^{85}$, et ne présentant ni traces de collage ni cassures fraîches, porte un morceau de l'estampille PATERN FE et, dans un décor à métopes, les jambes de l'Apollon D. 57, un tortillon mal venu et la partie inférieure gauche (une jambe et une masse indistincte, allongée et renflée : l'avant-bras droit et la main tenant la bourse) du Mercure D. 289. L'autre tesson, qui n'avait encore

४.1 Vases céramiques ornés..., vol. I, p. IV-v et 142-143.

85 L'enregistrement date de 1915 et l'inventaire signale qu'il s'agit d'un lot de pieces de la coll. Plicque ayant pour la plupart perdu leur numéro d'origine. C'est donc le fait que le lesson ait été extrait de la collection, comme d'autres objets signés, avant la date de l'enregistrement qui autorise à penser qu'il avait déjà attiré l'attention. 


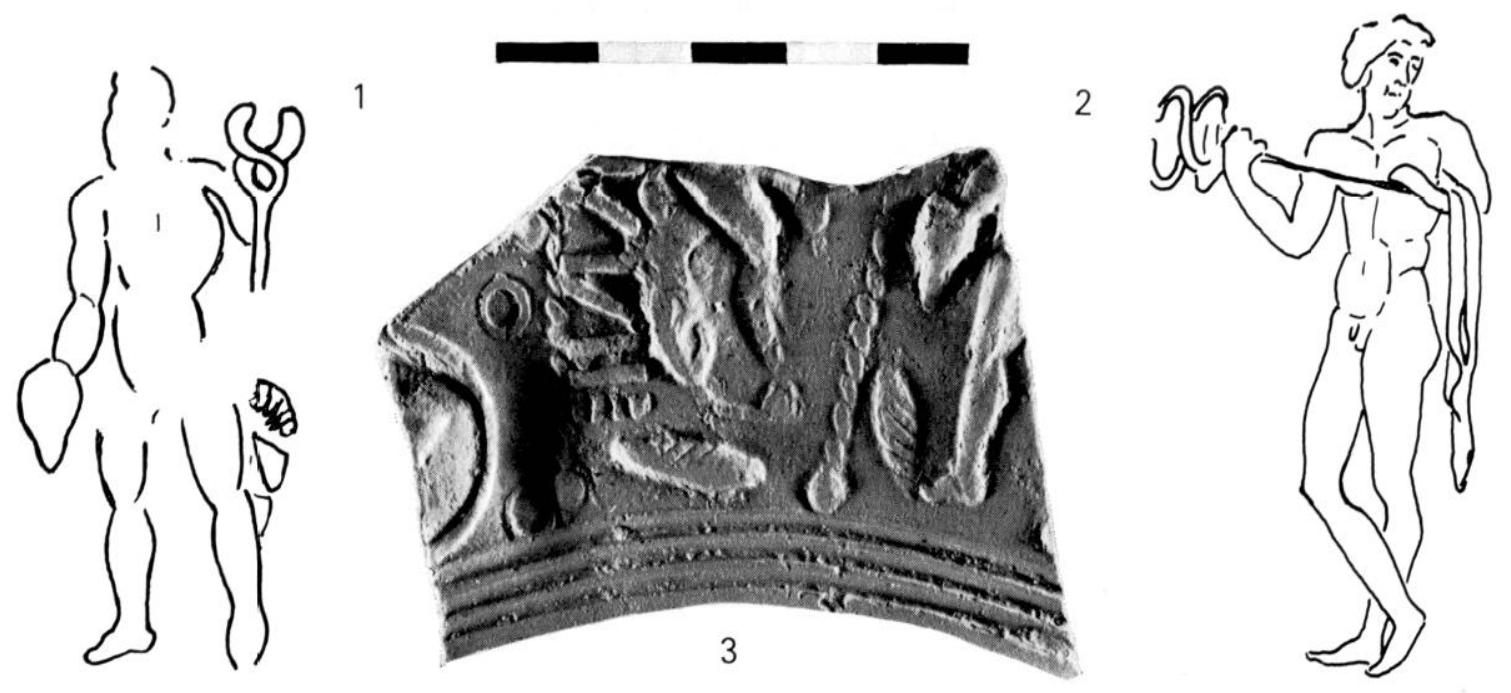

12 Le Vercure du gobelet signé. 1 : poinçon D. 289 d'apres Jes deux fragments du vase; 2 : poinçon D. 293 ; 3 : fragment recensé en 1915 sous le numéro 63327.

été ni inventorié, ni même nettoyé, conserve le reste du Vercure et une métope coupée à arceau. Déchelette n'a connu qu'un exemplaire signé ${ }^{86}$ : le vase $n^{0} 43$; nous ne connaissons qu'un échantillon estampillé : le fragment précédemment décrit. De deux choses l'une : ou il s'agit de deux gobelets différents - dont le premier s'est égaré depuis les travaux de Déchelette et dont le second, le seul que nous ayons. a échappé au premier inventaire ${ }^{87}$, ou il y a des inexactitudes dans la fiche reproduile sous le $n^{0} 43$. Le tesson 63327 et le vase ne seraient alors qu'un seul et même objet. Bien qu'il me soit impossible de trancher formellement et que je ne puisse apporter au débat que quelques observations, la seconde hypothèse ne me parait pas totalement dépourvue de vraisemblance.

Si le matéricl ćtudié à Lezoux par Déchelette ${ }^{88}$ a été dans sa totalité transporté à Saint-Germain, il est peu probable qu'un vase largement conservé et, qui plus est. estampillé ail disparu : j'ai constaté, en reprenant le classement que souhaitait l'illustre archéologue, que les tessons, surtout anonymes, ont connu diverses fortunes au cours des aménagements successifs du musée, mais que les pièces assez complètes et les exemplaires signés ont été, eux, l'objet d'une particulière attention. Par ailleurs, la différence entre le signalement du tesson 63327 et celui de gobelet $n^{0} 43$ tient, pour une part, à l'état du vase, pour l'autre, à l'identification d'un poinçon. Or, s'il est vrai que l'inventaire de Déchelette distingue, le plus souvent, pièces entières et fragments, une inexactitude n'est pas impossible et elle est tout à fait explicable, si l'on songe à l'abondance de la vaisselle recensée ${ }^{89}$.

$86 \quad$ O.c., vol. I, p. 173.

87 Cf. les commentaires de Déchelette sur le morcellement des vases et les dillicultés de l'enquête (o.c., vol. I p. v et 143, n. 1 .

88 En fait, certaines parties de la collection rassemblées par le docteur Plicque ne sont pas venurs à SaintGermain (cf. R.-E. Doraxio, La coll. Plicque al Musée des Antiquiles nationales, dans Mélanges Radel, Rev. él. anc., 1940, p. 614). Plusieurs lots sont connus en Angleterre (Durham, Nottingham;, an Hollande (musee de Leyde, an France (musée de Clermont-Ferrand, coll. Mathonnière-Plicque dans les Alpes-Maritimes, en particulier à Cannes). On ignore, d'autre part, si la fiche de Déchelette sur le vase $n^{\circ} 43$ de Paternus a été établie à I.c'zoux ou a SaintGermain el si, par conséquent, le gobelet risque de faire partie du materiel dispersé.

89 J'en ai relevé quelques-ınes, quand j'ai préparé l'inventaire des moules signés (Anliquilés nalionales, 4, 1972 , p. 63-82!, soil dans la description des décors, soit dans les références et l'illustration de quelques signatures. Dans le cas présent, seule la transcription complète de l'estampille pose un problème: : Déchelette, pour les poinçons figurés, ne donne que la référence au numéro de son catalogue, sans mentionner l'écat de conservation du décor. 
On remarquera, par ailleurs, que les décors énumérés : deux poinçons, dont un Apollon, ne permettent pas d'imaginer le reste de la frise, supposée en bon état. Mème si l'argument a silentio ne peut ètre tenu pour décisif ${ }^{90}$, on ne saurait considérer comme négligeable la coïncidence qui fait que trois poinçons - une signature et deux personnages - se trouvent sur le vase de I)échelette et que trois motifs analogues ont été isolés sur le tesson 63327 par une cassure ancienne. De plus, deux de ceux-ci : l'estampille et l'Apollon sont, dans les deux cas identiques. Reste l'identification du Mercure tantôt avec D. 293 (vase no 43), tantòt avec D. 289 (tesson 63327). Si l'on écarte, provisoirement, même sans l'exclure, la possibilité d'une simple erreur matérielle de fichier (comme il est facile d'en commettre), on peut se demander si l'état du Mercure sur le tesson - ì supposer que celui-ci fùt incomplet comme 63327 - ne favorisait pas une confusion : D. 293 représente le dieu de trois-quarts, à gauche, en appui sur une jambe, l'autre pliée et croisée devant la première, au niveau de la cheville et la forme globale du motif accidentellement constitué, sur le fragment 63327, par le tortillon indistinct et les restes, mal venus, du personnage n'est pas sans analogie avec la silhouette des jambes de D. 293 (fig. 12). Dans celte hypothèse, l'identification inexacte du poinçon s'expliquerait, comme la brièveté de la liste des décors du vase de Déchelette, par le fait que celui-ci n'aurait eu entre les mains qu'un spécimen incomplet. Une dernière remarque peut venir à l'appui de ces présomptions : si le gobelet no 43 portait un Vercure entier, pourquoi J. Déchelette a-t-il restitué le poinçon 293 à l'aide de deux dessins partiels exécutés à partir de fragments de moules et raccordés entre eux?

J'ai tenté, enfin, une contre-épreuve pour voir quelles chances avait le Mercure ID. 293 de se trouver sur un vase de P'alernus $I I$, supposé différent de l'exemplaire 63327. En fait, actuellement, l'attribution de ce poinçon au répertoire du potier semble se fonder exclusivement sur la notice $\mathrm{n}^{0} 43$ de Déchelette et n'être confirmée par aucun nouvel échantillon ${ }^{91}$, tandis que plusieurs pièces signées attestent, comme le tesson reconstitué 63327, l'emploi, dans cet atelier, du Mercure D. $289^{92}$. Ce n'est donc pas par ce biais que l'on peut, vu l'état actuel du répertoire attribué au potier, rendre probable l'existence du "vase» décrit par J. Déchelette.

Colette Bémont.

90 La description n'est pas toujours exhaustive et ne relient souvent que les principaux éléments de la frise.

91 F. Oswald (o.c., n ${ }^{0} 520$, ne se réfère qu'à la notice de Déchelelte. G. Simpson, d'autre part, a eu l'extrême amabilite de me confirmer que l'index de Central gaulish potlers (p. 287) reprenait l'information fournie par J. Déchelette et de m'indiquer qu'elle ne connaissait, pour sa part, aucun autre exemple de ce poinçon associé à cette estampille. Selon le I)r Simpson, il semble aussi que l'emplci du Mercure' I) 293 par Laxtucissa, associé probable ou prédécesseur de Paternus II, soit rare et qu'on l'observe plutòt sur des échantillons anépigraphes attribues au style de ce potier.

92 F. Oswald (o.c., n० 529) cite deux exemples signés : l'un au British Museum (= S.S., pl. 105, 18), l'autre à Cirencester ( $=$ S.S., pl. 108, 3.5). S.S. ajoutent un bol du Guildhall (pl. 105, 15).

N.B. Les clichés de l'illustration pholographique de cet article appartiennent à la photothèque du Musée des Antiquités nationales. 
II. Catalogue des pongçons

\begin{tabular}{|c|c|c|c|c|}
\hline Motifs & $\mathrm{N}^{0} \mathrm{r}$. & Attributions & RÉFÉRENCES & Motifs Associés \\
\hline 1 cordon torsadé & A 36 & $\begin{array}{l}\text { Albucius, Banuus, Iullinus, } \\
\text { Iustus, Mercator II, Pater- } \\
\text { nus II, Seuerus }\end{array}$ & & tous \\
\hline 2 petit arceau & & & & $1,11,15,18,22,25,29,51$ \\
\hline 3 grand arceau & & Paternus II & 63327 & $\begin{array}{r}1,8,11,12 ?, 15,16,19,22, \\
25,26 ?, 27,28,29,30,32 \\
33,35,38,39,40,42,44 \\
47,48 ?, 49,50,53 ?, 54\end{array}$ \\
\hline 4 petit double arceau & & & & $1,8,12,15,24,29,33,36$ \\
\hline 5 grand double arceau & & & & $\begin{array}{r}1,8,11,12,15,17,18,20,21 \\
22,25,29,30,31,33,35, \\
37,41,46,48,49,50,52\end{array}$ \\
\hline $\begin{array}{c}6{ }^{\circ} \text { double arceau outre- } \\
\text { passé }\end{array}$ & $F 63$ & Paternus II(s) & P 599 & $1,8,14,15,24,29,33,49$ \\
\hline 7 double arceau strié & $F 62$ & & & $1,8,15,21,29,31,42,47$ \\
\hline 8 petit anneau & & Paternus II & $\begin{array}{l}63327 ; \text { S.S. } \\
\quad 30,27\end{array}$ & $\begin{array}{l}1,3,4,5,6,7,11,12,15,16 \\
20,21,24,29,30,31,32 \\
33,35,36,38 ?, 39,40,41 \\
42,52,54\end{array}$ \\
\hline 9 anneau & & Paternus II & $66491(\mathrm{~A} 1)$ & $\begin{array}{l}1,10,11,15,16,22 \text { ou } 23 ? \\
24,34\end{array}$ \\
\hline 10 très petit cercle & & & & $\begin{array}{l}1,9,11,15,16,22 \text { ou } 23 ? \\
24,34\end{array}$ \\
\hline 11 petit cercle & & Paternus II & 63327 & $\begin{array}{l}1,3,5,8,9,10,12,15,16 \\
17,18,22 \text { ou } 23 ?, 24,25 \\
26,28,29,32,33,34,35 \\
39,40,48,49,51\end{array}$ \\
\hline 12 cercle moyen & & Paternus II(s) & PM 352 & $\begin{array}{l}1,3 ?, 4,5,8,11,15,17,18 \\
22,24,29,30,33,35,36 \\
37,42,43,45,47,48,49 \\
52,53\end{array}$ \\
\hline 13 grand cercle & & & & 1,32 \\
\hline 14 double cercle $*$ & & & & $1,6,15,24,49$ \\
\hline 15 pois & & Paternus II & 63327 & $\begin{array}{l}\text { tous sauf } 7,13,26,28,37 \text {, } \\
\quad 43,45\end{array}$ \\
\hline 16 rosette à 6 pétales & C 123 & $\begin{array}{l}\text { Censorinus, Laxtucissa, Pater- } \\
\text { nus II, Quintilianus }\end{array}$ & & $\begin{array}{r}1,3,8,9,10,11,15,22 \text { ou } \\
23 \text { ? } 24,26,28,29,32,33 \\
34,35,38,39,40,49,54\end{array}$ \\
\hline 17 rosette à 7 pétales & C 137 & Paternus II & $\begin{array}{l}\text { S.S. } \\
\text { fig. } 30,3\end{array}$ & $1,5,11,12,15,22,29,48$ \\
\hline 18 rosette à 8 pétales torses & E 66 & & & $\begin{array}{l}1,2,5,11,15,22,25,29,30 \\
\quad 33,51\end{array}$ \\
\hline 19 rosette à 11 ? pétales & E 77 & Paternus II & & $\begin{array}{l}1,3,15,19,25,29,30,44 \\
49,50\end{array}$ \\
\hline $\begin{array}{l}20^{\circ} \text { rosette à } 11 \text { pétales tor- } \\
\text { ses }\end{array}$ & E 78 & Paternus II (s) & $\begin{array}{l}\text { A.J., } 1973, \\
\quad 39\end{array}$ & $1,5,8,15,20,30,33,41$ \\
\hline 21 rosette à 12 pétales & $E 67$ & & & $\begin{array}{l}1,5,7,8,15,29,31,41 ? \\
\quad 42,47\end{array}$ \\
\hline 22 rosette à 13 pétales & $C 240$ & Paternus II & $\begin{array}{l}\text { musée de } \\
\text { Cannes }\end{array}$ & $\begin{array}{l}1,2,3,5,9 ?, 10 ?, 11,12,15 \\
16,17,18,24 ?, 25,28,29 \\
30,33,34 ?, 35,37,39,48 \\
49,51\end{array}$ \\
\hline 23 rosette à 14 pétales? & C 242 & Banuus, Paternus II & & $\begin{array}{l}1,9,10,11,15,16,34 \text { (asso- } \\
\text { ciations incertaines : l'em- } \\
\text { ploi de } C 242 \text { n'est pas sûr) }\end{array}$ \\
\hline
\end{tabular}




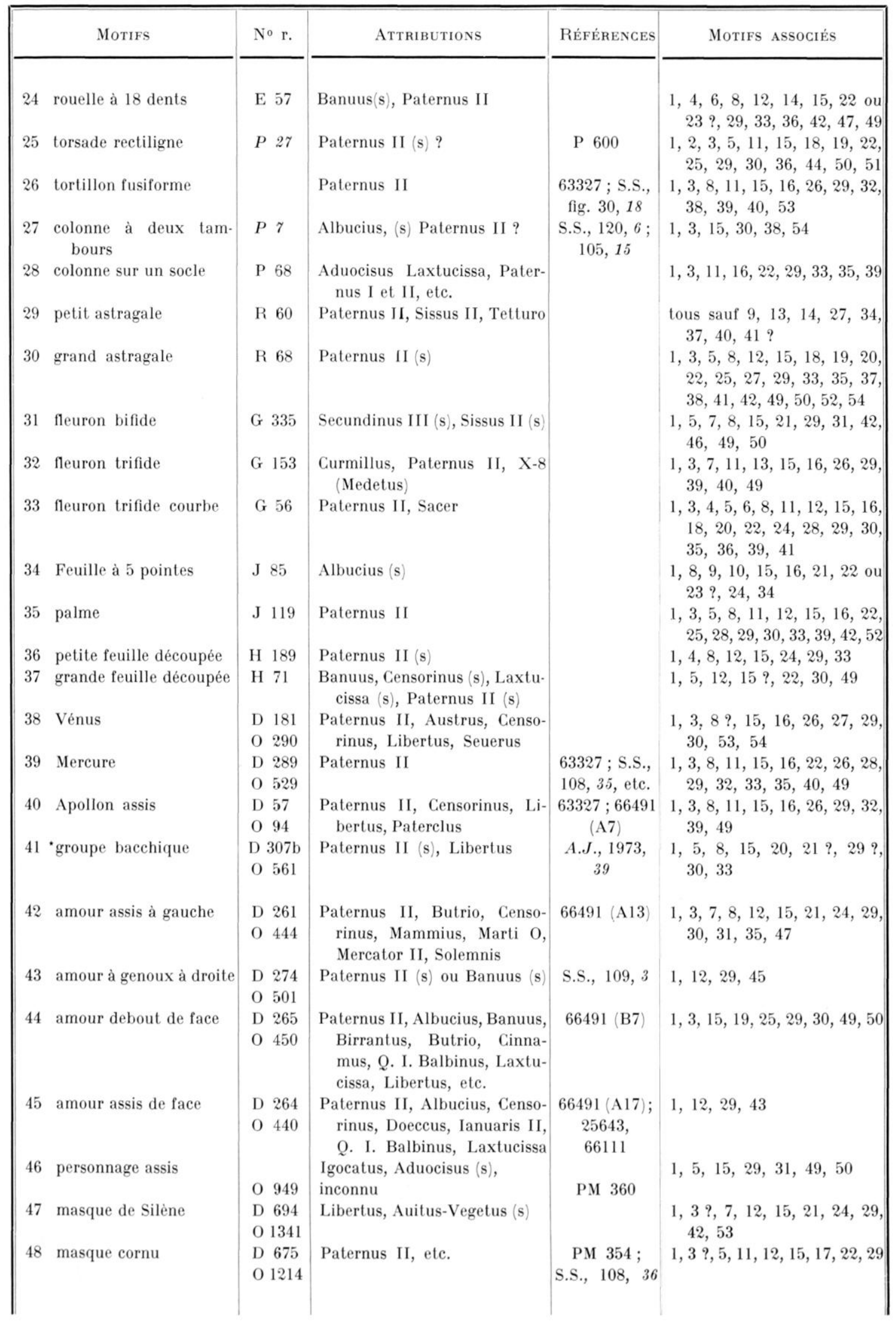




\begin{tabular}{|c|c|c|c|c|}
\hline Motifs & $\mathrm{N}^{0} \mathrm{r}$. & Attributions & RÉfÉrences & Motifs Associés \\
\hline 49 oiseau à droite & $\begin{array}{l}\text { D } 1010 \\
\text { O } 2316\end{array}$ & Paternus 1I, etc. & 63327 & $\begin{array}{l}1,3,5,6,11,12,14,15,16 \\
19,22,24,25,26,29,30 \\
31,32,37,39,40,44, \\
46,50\end{array}$ \\
\hline 50 oiseau à gauche & $\begin{array}{l}\text { D } 1011 \\
\text { O } 2324\end{array}$ & Paternus II, etc. & 14511 & $\begin{array}{l}1,3,5,15,19,25,29,30,31 \\
\quad 44,46,49\end{array}$ \\
\hline 51 oiseau stylisé à gauche & $\begin{array}{c}\mathrm{O} 2295 \\
\mathrm{~A}\end{array}$ & $\begin{array}{l}\text { Paternus II, Attianus (s), } \\
\text { Butrio (s), Iustus (s), Mer- } \\
\text { cator II }\end{array}$ & $\begin{array}{l}\text { cf. } 66491 \\
\text { (B1) }\end{array}$ & $1,2,11,15,18,22,25,29$ \\
\hline 52 coq à droite & $\begin{array}{l}\text { D } 1023 \\
\text { O } 2342\end{array}$ & $\begin{array}{l}\text { Paternus II (s), Catussa I, } \\
\text { Libertus, Paullus (s) }\end{array}$ & $\begin{array}{c}\text { P } 599 ; \\
\text { Ovilava, } 55,5\end{array}$ & $1,5,8,12,15,29,30,35$ \\
\hline $53^{*}$ poisson à gauche & $\begin{array}{l}\text { D } 1062 \\
\text { O } 2417\end{array}$ & $\begin{array}{l}\text { Paternus II (s), Butrio, Doec- } \\
\text { cus, Iustus, Libertus }\end{array}$ & $\begin{array}{l}\text { A.J., } \\
1957,9\end{array}$ & $1,3 ?, 12,15,26,29,38,47$ \\
\hline 54 sphinx à gauche & $\begin{array}{ll}\text { D } & 497 \\
\text { O } & 857\end{array}$ & $\begin{array}{l}\text { Paternus II, Cinnamus, Paul- } \\
\text { lus }\end{array}$ & 25643 & $1,3,8,15,16,27,29,30,38$ \\
\hline
\end{tabular}

Numéros de référence : pour les motifs secondaires (1-37) la numérotation est empruntée au catalogue de G. B. Rogers. En ilalique : les numéros ajoutés à ce catalogue.

Allributions : j'ai ajouté à l'inventaire des potiers déjà connus pour avoir employés certains poinçons, des attributions nouvelles à Paternus II fondées, en particulier, sur des inédits du M.A.X.

Références : ne figurent dans cette rubrique que la justification de nouvelles attributions à Palernus II et, pour les motifs figurés déjà attestès, la mention des pièces qui confirment l'emploi du même poinçon par ce potier (excepté pour le no 46 :

Motifs associés : liste limilée, pour chaque poinçon, au matériel fourni par nos vases at nos moules.

- Poincons attestés dans le style de Palernus $I I$ (en dehors des vases inédits ici publiés exclusivement sur des gobelets.

* Il n'est pas exclu que ce motif résulte de la combinaison de deux poincons. 